\title{
Instanton theory of ground-state tunneling splittings with general paths
}

Cite as: J. Chem. Phys. 152, 084111 (2020); https://doi.org/10.1063/1.5145278

Submitted: 16 January 2020 . Accepted: 11 February 2020 . Published Online: 26 February 2020

Mihael Eraković (D), Christophe L. Vaillant (D), and Marko T. Cvitaš (iD)
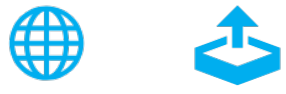

\section{ARTICLES YOU MAY BE INTERESTED IN}

Geometry optimization using Gaussian process regression in internal coordinate systems The Journal of Chemical Physics 152, 084112 (2020); https://doi.org/10.1063/1.5144603

The density matrix renormalization group in chemistry and molecular physics: Recent developments and new challenges

The Journal of Chemical Physics 152, 040903 (2020); https://doi.org/10.1063/1.5129672

Generalized spin mapping for quantum-classical dynamics

The Journal of Chemical Physics 152, 084110 (2020); https://doi.org/10.1063/1.5143412

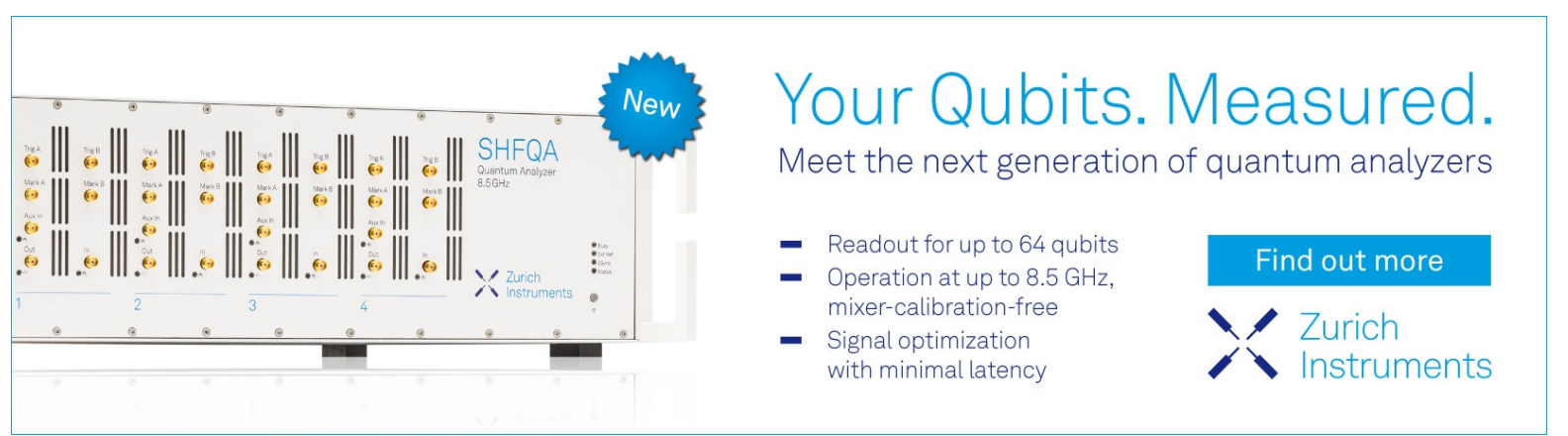




\title{
Instanton theory of ground-state tunneling splittings with general paths
}

\author{
Cite as: J. Chem. Phys. 152, 084111 (2020); doi: 10.1063/1.5145278 \\ Submitted: 16 January 2020 - Accepted: 11 February 2020 • \\ Published Online: 26 February 2020
}

Mihael Eraković, ${ }^{\text {(D) }}$ Christophe L. Vaillant, ${ }^{2}$ (D) and Marko T. Cvitaš

\begin{abstract}
AFFILIATIONS
${ }^{1}$ Department of Physical Chemistry, Ruđter Bošković Institute, Bijenička Cesta 54, 10000 Zagreb, Croatia

${ }^{2}$ Laboratory of Theoretical Physical Chemistry, Institut des Sciences et Ingénierie Chimiques, École Polytechnique Fédérale de Lausanne, CH-1015 Lausanne, Switzerland
\end{abstract}

a) Author to whom correspondence should be addressed: mcvitas@irb.hr

\begin{abstract}
We derive a multidimensional instanton theory for calculating ground-state tunneling splittings in Cartesian coordinates for general paths. It is an extension of the method by Mil'nikov and Nakamura [J. Chem. Phys. 115, 6881 (2001)] to include asymmetric paths that are necessary for calculating tunneling splitting patterns in multi-well systems, such as water clusters. The approach avoids multiple expensive matrix diagonalizations to converge the fluctuation prefactor in the ring-polymer instanton (RPI) method, and instead replaces them by an integration of a Riccati differential equation. When combined with the string method for locating instantons, we avoid the need to converge the calculation with respect to the imaginary time period of the semiclassical orbit, thereby reducing the number of convergence parameters of the optimized object to just one: the number of equally spaced system replicas used to represent the instanton path. The entirety of the numerical effort is thus concentrated in optimizing the shape of the path and evaluating hessians along the path, which is a dramatic improvement over RPI. In addition to the standard instanton approximations, we neglect the coupling of vibrational modes to external rotations. The method is tested on the model potential of malonaldehyde and on the water dimer and trimer, giving close agreement with RPI at a much-reduced cost.
\end{abstract}

Published under license by AIP Publishing. https://doi.org/10.1063/1.5145278

\section{INTRODUCTION}

In non-rigid molecular systems, the rovibrational energy levels can be split due to the presence of degenerate minima accessible by tunneling. ${ }^{1}$ These splittings vary over many orders of magnitude and, even for the ground states of molecules, are affected by the properties of the potential energy surface (PES) at far-fromequilibrium geometries. This makes the splittings difficult to calculate using exact variational methods, as basis sets need to span relatively large regions of configuration space, and a tight convergence of the basis is needed in order to resolve often tiny energy differences in the levels. The exponential scaling of variational methods with basis size thus leaves only small gas-phase systems ${ }^{2}$ amenable to accurate treatments.

Tunneling splittings in symmetric double-well systems, such as, for example, proton transfer in malonaldehyde ${ }^{3,4}$ and the vinyl radi$\mathrm{cal}^{5}{ }^{5}$ or the collective migration of hydrogen atoms in ammonia ${ }^{6}$ and hydronium, have been studied extensively. The splittings serve as benchmarks for studying the interactions at play, while the models of interactions serve as a testing ground for dynamical calculation methods of tunneling splittings. ${ }^{8-19}$ Studies of tunneling splitting patterns in water clusters have particularly come into focus, as the interactions and the rearrangements involved are important for the understanding of liquid water from first principles. ${ }^{20,21}$ These systems exhibit multiple degenerate wells. Only the water dimer can be treated by exact methods, ${ }^{22,23}$ whereas the calculation of splittings in larger clusters has to rely on approximations. These include various reduced-dimensionality ${ }^{24-28}$ and semiclassical methods. ${ }^{29,30}$ Early work on water clusters used diffusion Monte Carlo, ${ }^{31,32}$ which requires knowledge of the position of the nodal surface of the excited state. Alternatively, the WKB method has been employed in combination with group theory, ${ }^{33,34}$ whereby an a priori choice of the path connecting the minima has to be made, with the results being highly dependent on that choice. More recently, the semiclassical instanton method has been extended to the treatment of multi-well system ${ }^{30}$ and applied to a range of water clusters of different $\operatorname{sizes}^{35-37}$ in order 
to interpret the experimental splitting patterns. Possibly, the only method that can improve on the instanton results at the moment is the recently-developed path integral molecular dynamics method $(\mathrm{PIMD})^{38,39}$ that uses instanton trajectories ${ }^{40}$ as an input and comes at comparatively larger computational cost.

Instanton theory for the calculation of ground-state tunneling splittings was introduced by Vainshtein et al. ${ }^{41}$ The splittings are obtained from the quantum partition function, which, in the Feynman path integral formulation, ${ }^{42}$ is approximated using the dominant minimum action path (MAP) and the paths in its vicinity. Mil'nikov and Nakamura ${ }^{43}$ generalized the instanton method to multidimensional systems and turned it into a numerical tool. However, they used internal coordinates and, as they concentrated on applications in double-well systems, ${ }^{44}$ the method they developed assumed the symmetry of the MAP. Competing instanton approaches also developed in parallel to this. ${ }^{45,46} \mathrm{~A}$ more recent version of the instanton theory that works in Cartesian coordinates, the ring-polymer instanton (RPI) method, ${ }^{47}$ has been developed and extended to the treatment of multiple well systems. ${ }^{30}$ The multi-well splitting patterns often arise due to asymmetric instanton paths, ${ }^{30}$ where the atoms involved in the tunneling motion take on different roles in the degenerate minima they connect. Although the simplicity of the RPI method and the generality of using Cartesian coordinates are appealing, the Mil'nikov-Nakamura method [which we henceforth refer to as the Jacobi fields instanton (JFI)] remains computationally more efficient.

A numerical application of the RPI method ${ }^{47}$ consists of three time-consuming steps. In the first step, an optimization of the path (discretized into "beads" of the ring polymer) is carried out by minimizing the action integral. In the second step, the hessian for each bead is evaluated along the MAP. In the third step, the so-called fluctuation prefactor, ${ }^{4,49}$ which quantifies the contributions of harmonic fluctuations about the MAP, is calculated. This last step involves a diagonalization of a large banded matrix in the ring-polymer space, which needs to be repeated many times until a converged value is found. Difficulties with the convergence and the methods of improving it have already been studied and proposed. ${ }^{10,50}$ The efficient evaluation of the fluctuation prefactor is also important for instanton theories of dynamical quantities, such as temperature-dependent ${ }^{51,52}$ and energy-dependent reaction rates, ${ }^{53,54}$ where several methods have been proposed to speed up the RPI calculations. ${ }^{54-57}$

A numerically efficient instanton method is highly desirable to minimize the number of potential evaluations, thus allowing the calculation of splittings in larger systems or the use of more accurate on-the-fly electronic structure calculations for the potential. RPI calculations become inefficient if the imaginary time path is particularly long, as is the case for the water pentamer, ${ }^{37}$ where the skeletal motion of oxygens in the puckered ring structure accompanies flips and bifurcations of hydrogen bonds, or when many instanton calculations are required, such as for rotationally-excited state calculations. ${ }^{58}$ The number of minima in a cluster, and thus the number of instantons, also rises factorially with the number of equivalent atoms (even if the final number of these paths is low $^{37}$ ), and a faster instanton evaluation could help identify candidates for a more accurate evaluation by PIMD. ${ }^{40}$
In this paper, we extend the original JFI method ${ }^{43}$ to asymmetric paths. This extension, which we refer to as the Jacobi fields instanton for general paths (JFI-GP), greatly improves the efficiency of obtaining the fluctuation prefactor for the tunneling matrix elements compared to the RPI method, while retaining all of the RPI's advantages. In our approach, the MAP search that precedes the evaluation of the prefactor and the tunneling matrix element is carried out using either a string method ${ }^{10}$ with an limited-memory BroydenFletcher-Goldfarb-Shanno (LBFGS) optimizer ${ }^{59,60}$ or a quadratic string method. ${ }^{61,62}$ In contrast to the RPI method, the string optimization and the JFI calculations are done without reference to the imaginary time period of the orbit $\beta \hbar$, thus avoiding the issues of convergence with $N$ found in RPI for paths requiring large values of $\beta .^{50}$ Only the number of equally spaced beads $N$ used to represent the path needs to be specified. Once the shape of the path has been determined with sufficient accuracy, hessians are evaluated at each bead, and the fluctuation prefactor can be calculated in a comparatively negligible time by solving a differential Riccati equation. This procedure thus moves the convergence with respect to $\beta$ into the convergence parameters of the Riccati differential equation solver, and an adaptive step size control of the integrator efficiently controls the accuracy of the prefactor. The combination of algorithms we use to calculate the tunneling splittings greatly simplifies the instanton method, to the point where it is practically a black-box method.

The rest of the paper is arranged as follows. In Sec. II, we describe instanton theory for multiple wells. In Sec. III, the formula for the fluctuation prefactor based on the JFI method is derived for general paths. In Sec. IV, we carry out numerical tests to validate the method against the RPI results and a direct one-way integration method. Results are summarized in Sec. V. Atomic units are used throughout $(\hbar=1)$.

\section{MULTI-WELL INSTANTON THEORY}

The tunneling spectrum of a system with $G$ degenerate wells can be obtained from the ratio of partition functions of the system with and without the inclusion of tunneling, $Q$ and $Q_{0}$, respectively, in the limit of zero temperature, as

$$
\lim _{\beta \rightarrow \infty} \frac{Q(\beta)}{Q_{0}(\beta)}=\frac{1}{G} \sum_{v=1}^{G} \mathrm{e}^{-\beta\left(E_{v}-E_{0}\right)},
$$

where $\beta=1 / k_{\mathrm{B}} T$ is the inverse temperature, $k_{\mathrm{B}}$ is the Boltzmann constant, $E_{v}$ is the energy of the $v$ th state in the full tunneling system, and $E_{0}$ is the zero-point energy in the absence of tunneling. The ratio in Eq. (1) can also be expressed via the tunneling matrix $\mathbf{W}$,

$$
\lim _{\beta \rightarrow \infty} \frac{Q(\beta)}{Q_{0}(\beta)}=\frac{1}{G} \operatorname{Tr}\left[\mathrm{e}^{-\beta \mathbf{W}}\right],
$$

whose rows and columns denote the different degenerate minima connected by the tunneling matrix elements.

The partition function in Eqs. (1) and (2) can be expressed in the discretized path-integral (ring-polymer) formalism using a sum over periodic orbits of imaginary time period $\beta$ as ${ }^{42}$

$$
\begin{aligned}
Q(\beta) & =\operatorname{Tr}\left[\mathrm{e}^{-\beta \hat{H}}\right] \\
& =\lim _{N \rightarrow \infty} \frac{1}{\sqrt{2 \pi \beta_{N}}} \int \ldots \int \prod_{i} \mathrm{~d} \mathbf{x}_{i} \mathrm{e}^{-S\left(\mathbf{x}_{i}\right)},
\end{aligned}
$$


where $S$ is the Euclidean action. The system is described using $N$ beads, or system replicas, separated by imaginary time steps of length $\beta_{N}=\beta / N$, where we use mass-scaled Cartesian coordinates $x_{i, j}=\sqrt{m_{j}} \times$ (position of atom $\left.j\right)$. Here, the first subscript labels the ring-polymer bead, the second subscript $j=1, \ldots, f$ labels the degree of freedom, and $f=3 \times$ (number of atoms) is the dimensionality of the system. Instanton theory ${ }^{1,41}$ approximates the exact expression in Eq. (3) by expanding the Euclidean action in the exponent to second order in $\mathbf{x}$ about the MAP. The instanton tunneling matrix element obtained in this way ${ }^{47,52}$ is given as

$$
h=-\frac{1}{\Phi} \sqrt{\frac{S_{0}}{2 \pi}} \mathrm{e}^{-S_{0}}
$$

expressed in terms of the tunneling MAP that connects two minima, associated with the indices of the tunneling matrix $\mathbf{W}$. The action of the MAP is $S_{0}$, whereas the fluctuation prefactor $\Phi$ incorporates the contribution of nearby paths in a harmonic approximation using hessians evaluated along the MAP.

In a numerical implementation of the instanton method, ${ }^{52,63}$ we first need to locate all simple, single-barrier MAPs that connect pairs of degenerate minima. Within the RPI method, ${ }^{47}$ this is achieved by minimizing the action with respect to bead positions $\mathbf{x}$ for each value of the imaginary time period $\beta$ and the number of beads $N$. The imaginary time period $\beta$ needs to be large enough for a classical orbit to connect the two minima and $N$ large enough to faithfully represent the orbit. The optimized object is therefore defined by two parameters, and the converged paths exhibit a significant clustering of beads near the minima, where the instanton speed is low.

In the JFI approach, ${ }^{10,61,64}$ we determine the shape of MAPs by minimizing the Jacobi action ${ }^{65}$ without reference to the imaginary time,

$$
S_{\mathrm{J}}=\int_{\mathrm{x}(-\infty)}^{\mathrm{x}(+\infty)} p d x
$$

where $p=\dot{x}=\sqrt{2 V(x)}$ is the mass-scaled system momentum (speed) at zero energy, or equivalently, at zero temperature, and $V(x)$ is the PES with minima at $x( \pm \infty)$, which define the zero of the potential. The path is discretized using a string of equally spaced beads in mass-scaled coordinate space, instead of a ring polymer with beads equally spaced in imaginary time. This greatly reduces the number of beads $N$ required for the convergence. Minimization of the Jacobi action in $(N \times f)$-dimensional space is carried out using a standard gradient-based search method, such as the LBFGS ${ }^{59,60}$ or quadratic string ${ }^{61,62}$ methods, with the end bead geometries fixed at two minima, while their orientation is adjusted explicitly using quaternions. The only convergence parameter of the optimized object is $N$. According to the principle of least action, the dominant orbit is a classical imaginary time trajectory on the inverted potential at zero energy. The imaginary time dependence of the classical trajectory can thus be obtained through quadrature after the shape of the orbit has been determined.

With RPI, the prefactor $\Phi$ in Eq. (4) is evaluated from the hessians of the discretized action (in time- and mass-scaled coordinates),

$$
\mathbf{H}_{i, j ; i^{\prime}, j^{\prime}}=\frac{\partial^{2} S_{0}}{\partial\left(\sqrt{\beta_{N}} x_{i, j}\right) \partial\left(\sqrt{\beta_{N}} x_{i^{\prime}, j^{\prime}}\right)},
$$

where the hessians of the potential in Eq. (6) have been evaluated at each bead. Using the eigenvalues of Eq. (6) of the full system, $\lambda_{l}$, and the non-tunneling system eigenvalues $\lambda_{l}^{0}$, the ring-polymer approximation to the prefactor is given by

$$
\Phi=\left[\frac{\prod_{l} \lambda_{l}}{\prod_{l^{\prime}} \lambda_{l^{\prime}}^{0}}\right]^{1 / 2} .
$$

The eigenvalues of the overall rotations, translations, and the instanton velocity vector along the pathway are zero and have been excluded from the products in Eq. (7). The method thus requires a diagonalization of an $(N \times f)$-dimensional matrix. Although the matrix, Eq. (6), is banded, with a width of $2 f+1$ elements, the fluctuation prefactor $\Phi$ needs to be converged with respect to $\beta$ and $N$, and the diagonalization must therefore be repeated many times. For large systems (i.e., large $f$ ) or systems which involve slow motions near minima (i.e., large $\beta$ and $N$ ) caused by, e.g., the movement of heavy atoms, this step becomes a computational bottleneck.

In the last step of the multi-well instanton method, the pathways with non-negligible tunneling matrix elements are inserted into the tunneling matrix $\mathbf{W}$, including only a closed set of permutation-inversion operations, forming a molecular symmetry group that relate the accessible minima. The eigenvalues of the tunneling matrix give the tunneling spectrum, and the eigenvectors determine the symmetry of each level, labeled by the irreducible representations of the molecular symmetry group. Statistical weights of energy levels and the allowed transitions can then be obtained using the nuclear spin state symmetries by standard group theory methods. ${ }^{66}$ In the case of a double well potential, the splitting is just $-2 h$. The above treatment neglects the overall rotation of the molecule and the anharmonicity of the potential perpendicular to the instanton tunneling path.

\section{DERIVATION OF FLUCTUATION PREFACTOR FOR GENERAL PATHS}

An alternative approach to RPI is the JFI method, ${ }^{43}$ where $\Phi$ is evaluated by means of Jacobi fields. The determination of $\Phi$ reduces to the solution of a Riccati differential equation with negligible computational cost in comparison to matrix diagonalizations. Here, we extend this method to include asymmetric paths that regularly appear in molecular systems with multiple wells.

The fluctuation prefactor $\Phi$ in Eq. (7) can be expressed as a ratio of determinants of Schrödinger operators with Dirichlet boundary conditions at imaginary time $t= \pm \infty$. ${ }^{48,49}$ The ratio of determinants is related to the ratio of Jacobi fields $\mathbf{J}(t)$ defined along the instanton trajectory $^{48,49}$ as

$$
\begin{aligned}
\Phi & =\left[\frac{\operatorname{det}\left(-\partial_{t}^{2}+\mathbf{H}(t)\right)}{\operatorname{det}\left(-\partial_{t}^{2}+\mathbf{H}_{0}\right) \lambda}\right]^{1 / 2} \\
& =\left[\frac{\operatorname{det} \mathbf{J}\left(T_{2}\right)}{\operatorname{det} \mathbf{J}_{0}\left(T_{2}\right) \lambda\left(T_{2}\right)}\right]^{1 / 2},
\end{aligned}
$$

where the trajectory starts at a large negative time $-T_{1}$ at minimum 1 and runs to a large positive time $T_{2}$ at minimum 2 . Here, $\lambda$ is the eigenvalue that corresponds to the instanton velocity or imaginary time translation (which tends to zero in the limit of infinite $T_{1,2}$ ), 
$\mathbf{H}(t)$ is the hessian of the potential at $\mathbf{x}(t)$, and $\mathbf{H}_{0}$ is the hessian at the minima. The Jacobi fields $\mathbf{J}$ and $\mathbf{J}_{0}$ are $f \times f$ matrices and satisfy the coupled second-order matrix differential equations,

$$
\begin{aligned}
& \left(-\partial_{t}^{2}+\mathbf{H}(t)\right) \mathbf{J}(t)=\mathbf{0}, \\
& \left(-\partial_{t}^{2}+\mathbf{H}_{0}\right) \mathbf{J}_{0}(t)=\mathbf{0},
\end{aligned}
$$

with initial conditions,

$$
\begin{aligned}
& \mathbf{J}\left(-T_{1}\right)=\mathbf{J}_{0}\left(-T_{1}\right)=\mathbf{0}, \\
& \dot{\mathbf{J}}\left(-T_{1}\right)=\dot{\mathbf{J}}_{0}\left(-T_{1}\right)=\mathbf{I} .
\end{aligned}
$$

The ratio of the determinants and, correspondingly, of the Jacobi fields in Eq. (8) can be factorized into two parts. ${ }^{43}$ One part is onedimensional along the instanton trajectory and contains the singularity associated with the zero mode $\lambda$ of instanton velocity. The other part is $(f-1)$-dimensional in the space orthogonal to the instanton. ${ }^{43}$ The prefactor is then

$$
\Phi=\left[\frac{J^{\|}\left(T_{2}\right)}{J_{0}^{\|}\left(T_{2}\right) \lambda\left(T_{2}\right)}\right]^{1 / 2}\left[\frac{\operatorname{det} \mathbf{J}^{\perp}\left(T_{2}\right)}{\operatorname{det} J_{0}^{\perp}\left(T_{2}\right)}\right]^{1 / 2},
$$

where $J^{\|}$and $\mathbf{J}^{\perp}$ are Jacobi fields in the one- and $(f-1)$-dimensional projected spaces, respectively. The Jacobi fields also satisfy the equations of the same form given by Eq. (9), but with modified hessian matrices $\mathbf{H}$. The form of matrix $\mathbf{H}^{\perp}$ is given in Ref. 43 [see Eq. (43)] in terms of the hessian in the local $(f-1)$-dimensional coordinate system orthogonal to the instanton, and the curvature of the instanton trajectory. The hessian $H^{\|}$is the second derivative of the potential $V(x(t))$ with respect to $x$, the arc length distance from minimum 1 along the instanton. The axes of the local coordinate system are chosen to coincide with the normal modes at minimum 1 and are obtained elsewhere along the instanton by parallel transport (the change in any of the $f-1$ perpendicular axes unit vectors along the instanton trajectory remains parallel to the instanton ${ }^{43}$ ).

The Jacobi field $\mathbf{J}_{0}=J_{0}^{\|} \mathbf{J}_{0}^{\perp}$ at minimum 1 in Eq. (11) can be obtained from Eq. (9) subject to the initial condition Eq. (10), resulting in

$$
\mathbf{J}_{0}(t)=\mathbf{\Omega}_{0}^{-1} \sinh \boldsymbol{\Omega}_{0}\left(t+T_{1}\right),
$$

with $\mathbf{H}_{0}=\mathbf{\Omega}_{0}^{2}$. At large $t=T_{2}$, the determinant of the Jacobi field $\mathbf{J}_{0}$ is

$$
\operatorname{det} \mathbf{J}_{0}\left(T_{2}\right)=\frac{\exp \left[\operatorname{Tr} \Omega_{0}\left(T_{1}+T_{2}\right)\right]}{\operatorname{det} 2 \mathbf{\Omega}_{0}},
$$

where Eq. (C1) has been used.

The Jacobi field $J^{\|}$for the one-dimensional problem in Eq. (9) with $H^{\|}=V^{\prime \prime}(x(t))$ is obtained by first noting that one solution of the Jacobi equation is the instanton velocity $\eta=p \equiv \dot{x}$. The instanton trajectory approaches minima 1 and 2 along normal modes having frequencies $\omega_{1}$ and $\omega_{2}$, respectively, which are not necessarily equal for asymmetric paths. The asymptotic behavior of the solution $\eta$ in imaginary time near the minima is given by

$$
\begin{aligned}
\eta\left(-T_{1}\right) & =P \mathrm{e}^{-\omega_{1} T_{1}}, \\
\eta\left(T_{2}\right) & =Q \mathrm{e}^{-\omega_{2} T_{2}},
\end{aligned}
$$

for large $T_{1}$ and $T_{2}$. The proportionality constants $P$ and $Q$ are chosen such that the solution of the Jacobi equation $\eta$ coincides with the instanton speed. Appendix A shows how they can be obtained from the potential curve along the instanton trajectory.

The second linearly independent solution $\xi$ is obtained by requiring that the Wronskian $W=\eta \dot{\xi}-\dot{\eta} \xi=1$ (or through D'Alembert's construction ${ }^{48}$ ). The asymptotic behavior of this solution is therefore

$$
\begin{gathered}
\xi\left(-T_{1}\right)=-\frac{1}{2 \omega_{1} P} \mathrm{e}^{\omega_{1} T_{1}}, \\
\xi\left(T_{2}\right)=\frac{1}{2 \omega_{2} Q} \mathrm{e}^{\omega_{2} T_{2}} .
\end{gathered}
$$

The linear combination that satisfies the initial conditions Eq. (10) is

$$
J^{\|}(t)=-\xi\left(-T_{1}\right) \eta(t)+\eta\left(-T_{1}\right) \xi(t) .
$$

When evaluated at $t=T_{2}$, this gives

$$
J^{\|}\left(T_{2}\right)=\frac{1}{2 \omega_{1}} \frac{Q \mathrm{e}^{-\omega_{2} T_{2}}}{P \mathrm{e}^{-\omega_{1} T_{1}}}+\frac{1}{2 \omega_{2}} \frac{P \mathrm{e}^{-\omega_{1} T_{1}}}{Q \mathrm{e}^{-\omega_{2} T_{2}}},
$$

where we have used Eqs. (14) and (15).

We turn our attention now to the ratio $\eta\left(-T_{1}\right) / \eta\left(T_{2}\right)$ and its inverse that appears in Eq. (17). The limits of large $T_{1}$ and $T_{2}$ can be taken simultaneously, in such a way that the ratio evaluates to 1 . For a classical trajectory in the inverted potential at a small negative energy $E$, this condition is equivalent to requiring that the turning points of the trajectory are at the same energy, $V\left(x\left(-T_{1}\right)\right)=V\left(x\left(T_{2}\right)\right)=E$, as the energy $E$ is varied toward $E=0$. If $\omega_{1}=\omega_{2}$, we obtain $J=1 / \omega_{1}$, as in the symmetric path case. ${ }^{43}$ Consequently, $J^{\|}\left(T_{2}\right)$ remains finite. The explicit expression for the exponentially small eigenvalue $\lambda$ in Eq. (8) associated with the instanton velocity, for large but finite $T_{1}$ and $T_{2}$, is derived in Appendix B. Again, by taking the infinite-time limit in Eq. (B5) as above, the exponentially growing terms in $J_{0}^{\|}$and $\lambda$ cancel and a finite ratio of Jacobi fields in Eq. (11) in the direction along the instanton is obtained. Nevertheless, we do not take these limits in order to obtain the expression for $\Phi$ in terms of convergent factors in which the $T_{1}$ and $T_{2}$ limits can be taken separately.

Using Eqs. (B5) and (17), we obtain

$$
\frac{\lambda}{J^{\|}\left(T_{2}\right)}=\frac{\left(2 \omega_{1}\right)\left(2 \omega_{2}\right)\left(P \mathrm{e}^{-\omega_{1} T_{1}}\right)\left(\mathrm{Qe}^{-\omega_{2} T_{2}}\right)}{S_{0}},
$$

which forms a part of Eq. (11). In the numerator of the right-hand side of Eq. (18), we again recognize $\eta\left(-T_{1}\right)$ and $\eta\left(T_{2}\right)$. The two velocities are related via Eq. (A3) (where $A=-T_{1}$ and $B=T_{2}$ ), so that the right-hand side of Eq. (18) can be rearranged into the expression

$$
\frac{\lambda}{J^{\|}\left(T_{2}\right)}=\frac{\left(2 \omega_{1}\right)\left(2 \omega_{2}\right)\left(Q^{2} \mathrm{e}^{-2 \omega_{2} T_{2}}\right)}{S_{0}} \exp \left[-\int_{-T_{1}}^{T_{2}} \frac{\partial p}{\partial x} d t\right],
$$

which we will use below. We note now that contained within the integral in Eq. (19) is the log derivative of $\eta(t)$, namely $\dot{\eta} / \eta=d p / d x$, where $\eta(t)$ is the solution of the Jacobi equation along the instanton with initial condition $\dot{\eta} / \eta=\omega_{1}$.

The remaining quantity to be determined in Eq. (11) is the determinant of the Jacobi field $\mathbf{J}^{\perp}$. We will again accomplish this 
by integration of the associated Jacobi equation. Since $\mathbf{J}^{\perp}$ is exponentially large everywhere except in the vicinity of minimum 1 , it is more convenient to transform the Jacobi equation, Eq. (9), into a Riccati equation for the log derivative of the Jacobi field $\mathbf{A}=\mathbf{J J}^{-1}$ as

$$
\frac{d \mathbf{A}(t)}{d t}=\mathbf{H}(t)-\mathbf{A}^{2}(t) .
$$

We effectively integrate the exponents of the solutions of the Jacobi equation in imaginary time $t$. In the present case, the hessian is $\mathbf{H}^{\perp}(t)=\left(\boldsymbol{\Omega}^{\perp}(t)\right)^{2}$ and $\mathbf{A}^{\perp}=\dot{\boldsymbol{\eta}}^{\perp} / \boldsymbol{\eta}^{\perp}$. The initial condition, Eq. (10), implies an infinite initial $\mathbf{A}^{\perp}$ at minimum 1 . In fact, only one stable solution can be propagated outward from minima, specifically, the one with the initial condition $\mathbf{A}^{\perp}(-\infty)=\boldsymbol{\Omega}_{1}^{\perp}$. Due to the infinite time spent in the vicinity of the minima, only the exponentially growing terms, $\exp \left(\Omega_{1}^{\perp}\left(t+T_{1}\right)\right)$, survive [see Eq. (12)]. From Eqs. (12) and (13) (or through D'Alembert's construction ${ }^{43,48}$ ), it can be deduced that the required determinant of $\mathbf{J}^{\perp}$ is related to the stable solution $\boldsymbol{\eta}^{\perp}$ by

$$
\operatorname{det} \mathbf{J}^{\perp}\left(T_{2}\right)=\frac{\operatorname{det} \boldsymbol{\eta}^{\perp}\left(T_{2}\right)}{\operatorname{det} 2 \Omega_{1}^{\perp}},
$$

where $\boldsymbol{\eta}^{\perp}\left(-T_{1}\right)=\mathbf{I}, \dot{\boldsymbol{\eta}}^{\perp}\left(-T_{1}\right)=\boldsymbol{\Omega}_{1}^{\perp}$, and $\operatorname{det} \boldsymbol{\Omega}_{0}=\omega_{1} \operatorname{det} \boldsymbol{\Omega}_{1}^{\perp}$. The determinant of $\boldsymbol{\eta}^{\perp}$ is obtained from its log derivative using Eq. (C4), subject to the above initial conditions,

$$
\operatorname{det} \boldsymbol{\eta}^{\perp}(t)=\exp \left[\int_{-T_{1}}^{t} \operatorname{Tr} \mathbf{A}^{\perp}\left(t^{\prime}\right) d t^{\prime}\right]
$$

where $\mathbf{A}^{\perp}(t)$ is obtained by solving the Riccati equation, Eq. (20).

We can now insert Eqs. (13), (19), (21), and (22) in Eq. (11) and group all the exponentials together to obtain

$$
\begin{aligned}
\frac{1}{\Phi^{2}}= & \frac{\left(2 \omega_{2}\right) Q^{2}}{S_{0}} \exp \left[\int_{-T_{1}}^{T_{2}} \operatorname{Tr} \boldsymbol{\Omega}_{0}^{\prime}-\left(\frac{\partial p}{\partial x}+\operatorname{Tr} \mathbf{A}^{\perp}\left(t^{\prime}\right)\right) d t^{\prime}\right] \\
& \times \frac{\left(2 \omega_{1}\right) \operatorname{det} 2 \boldsymbol{\Omega}_{1}^{\perp}}{\operatorname{det} 2 \boldsymbol{\Omega}_{0}},
\end{aligned}
$$

where $\operatorname{Tr} \boldsymbol{\Omega}_{0}^{\prime}=\operatorname{Tr} \boldsymbol{\Omega}_{0}$ for $t \in\left(-T_{1}, 0\right)$ and $\operatorname{Tr} \boldsymbol{\Omega}_{0}^{\prime}=\operatorname{Tr} \boldsymbol{\Omega}_{0}-2 \omega_{2}$ for $t \in\left(0, T_{2}\right)$. The last factor on the right-hand side of Eq. (23) evaluates to one. Since $A^{\|}=d p / d x$, the expression in the parentheses inside the integral in Eq. (23) equals the trace of $\mathbf{A}$, i.e., $\operatorname{Tr} \mathbf{A}=A^{\|}+\operatorname{TrA}^{\perp} .^{43}$ Local coordinates are connected to Cartesian coordinates through an orthogonal transformation, ${ }^{43}$ and we are free to use any coordinate system to evaluate Eq. (23). The final expression for the tunneling matrix element connecting minima 1 and 2 is obtained by taking limits of infinite $T_{1,2}$ as

$$
h_{12}=-\sqrt{\frac{\omega_{2}}{\pi}} \mathrm{e}^{-S_{0}} Q \exp \left[\frac{1}{2} \int_{-\infty}^{\infty}\left(\operatorname{Tr} \boldsymbol{\Omega}_{0}^{\prime}-\operatorname{Tr} \mathbf{A}\left(t^{\prime}\right)\right) d t^{\prime}\right],
$$

where $\mathbf{A}(t)$ is obtained by solving Riccati equation (20) with the initial condition $\mathbf{A}(-\infty)=\boldsymbol{\Omega}_{0}$. The calculation of $Q$ is achieved using Eq. (A5), as described in Appendix A. The integral in Eq. (24) converges at minimum 1 , as seen from the initial condition, and at minimum 2 because $\mathbf{A}^{\perp}$ coincides with the hessian perpendicular to the instanton (the mode with frequency $\omega_{2}$ ), ${ }^{43}$ as exponentially growing terms become dominant, while $d p / d x$ tends to $-\omega_{2}$. Equation (24) is also independent of the position of time origin $t=0$, as any shift of the origin that affects $\operatorname{Tr} \Omega_{0}^{\prime}$ in Eq. (24) will be compensated by the value of $Q$ [see Eq. (A4)]. Note that $\operatorname{Tr} \mathbf{A}(t)$ is asymmetric even for symmetric paths.

Due to the infinite time that the instanton trajectory spends near minima, it is not possible to numerically integrate the Riccati equation in their vicinity. At the start of the integration, we jump over the singularity at minimum 1 as described in Appendix D. However, in order to avoid the inherent instability at the other end of the integration, a different approach is needed, which we now derive.

As discussed above, $\boldsymbol{\eta}^{\perp}$ is the only stable solution near minimum 1 (in the space that is orthogonal to the instanton trajectory). Analogously, we now define $\tilde{\boldsymbol{\eta}}^{\perp}$, with the initial conditions $\tilde{\boldsymbol{\eta}}^{\perp}\left(T_{2}\right)=\mathbf{I}$ and $\dot{\tilde{\boldsymbol{\eta}}}^{\perp}\left(T_{2}\right)=-\boldsymbol{\Omega}_{2}^{\perp}$, the only stable solution that can be propagated in the negative time direction from minimum 2 toward minimum 1. We drop the " $\perp$ " symbol for $\boldsymbol{\eta}$ below to avoid clutter and recast the Jacobi equations in Eq. (9) for $\boldsymbol{\eta}$ and $\tilde{\boldsymbol{\eta}}$ into the first order matrix differential equation,

$$
\frac{d}{d t}\left(\begin{array}{cc}
\boldsymbol{\eta} & \tilde{\boldsymbol{\eta}} \\
\dot{\boldsymbol{\eta}} & \dot{\boldsymbol{\eta}}
\end{array}\right)=\left(\begin{array}{cc}
\mathbf{0} & \mathrm{I} \\
\left(\mathbf{\Omega}^{\perp}(t)\right)^{2} & \mathbf{0}
\end{array}\right)\left(\begin{array}{ll}
\boldsymbol{\eta} & \tilde{\boldsymbol{\eta}} \\
\dot{\boldsymbol{\eta}} & \dot{\tilde{\boldsymbol{\eta}}}
\end{array}\right) .
$$

Equation (25) is of the form $\dot{\mathbf{Y}}(t)=\mathbf{B}(t) \mathbf{Y}(t)$ and its infinitesimal time evolution is given by

$$
\mathbf{Y}(t+d t)=\mathrm{e}^{\mathbf{B} d t} \mathbf{Y}(t)
$$

We now take the determinant of both sides of Eq. (26) and use Eq. (C1) to obtain $\operatorname{det} \mathbf{Y}(t+d t)=\operatorname{det} \mathbf{Y}(t)$. Alternatively, we can use Eq. (C4) to calculate the time derivative of $\operatorname{det} \mathbf{Y}$ as

$$
\frac{d}{d t} \operatorname{det} \mathbf{Y}(t)=\operatorname{det} \mathbf{Y}(t) \operatorname{Tr} \mathbf{B}(t)
$$

where $\mathbf{B}$ is the $\log$ derivative of $\mathbf{Y}, \mathbf{B}=\dot{\mathbf{Y}} \mathbf{Y}^{-1}$. From Eq. (25), it is clear that $\operatorname{Tr} \mathbf{B}=0$ by construction, which proves that det $\mathbf{Y}(t)=$ const, and that the Bs in Eqs. (26) and (27) are identical.

We now proceed to evaluate the determinant of $\mathbf{Y}$, which is time independent, at $-T_{1}$, at $T_{2}$ and at a general time $t$ in-between, as

$$
\begin{array}{r}
\operatorname{det} \mathbf{Y}\left(-T_{1}\right)=\left|\begin{array}{cc}
\mathbf{I} & \tilde{\boldsymbol{\eta}}\left(-T_{1}\right) \\
\mathbf{\Omega}_{1}^{\perp} & -\boldsymbol{\Omega}_{1}^{\perp} \tilde{\boldsymbol{\eta}}\left(-T_{1}\right)
\end{array}\right|=(-2)^{f-1} \operatorname{det} \boldsymbol{\Omega}_{1}^{\perp} \operatorname{det} \tilde{\boldsymbol{\eta}}\left(-T_{1}\right), \\
\operatorname{det} \mathbf{Y}\left(T_{2}\right)=\left|\begin{array}{cc}
\boldsymbol{\eta}\left(T_{2}\right) & \mathbf{I} \\
\boldsymbol{\Omega}_{2}^{\perp} \boldsymbol{\eta}\left(T_{2}\right) & -\mathbf{\Omega}_{2}^{\perp}
\end{array}\right|=(-2)^{f-1} \operatorname{det} \boldsymbol{\Omega}_{2}^{\perp} \operatorname{det} \boldsymbol{\eta}\left(T_{2}\right), \\
\operatorname{det} \mathbf{Y}(t)=\left|\begin{array}{cc}
\boldsymbol{\eta}(t) & \tilde{\boldsymbol{\eta}}(t) \\
\dot{\boldsymbol{\eta}}(t) & \dot{\tilde{\boldsymbol{\eta}}}(t)
\end{array}\right|=\operatorname{det} \boldsymbol{\eta}(t) \operatorname{det}\left(\tilde{\mathbf{A}}^{\perp}(t)-\mathbf{A}^{\perp}(t)\right) \operatorname{det} \tilde{\boldsymbol{\eta}}(t),
\end{array}
$$

where we have used Eq. (C2) and the fact that exponentially growing terms eventually dominate in the (harmonic) vicinity of the minima at $-T_{1}$ and $T_{2}$. By equating Eqs. (28) and (29), and from Eq. (21), we have that

$$
\operatorname{det} \mathbf{J}^{\perp}\left(T_{2}\right)=\frac{\operatorname{det} \boldsymbol{\eta}^{\perp}\left(T_{2}\right)}{\operatorname{det} 2 \mathbf{\Omega}_{1}^{\perp}}=\frac{\operatorname{det} \tilde{\boldsymbol{\eta}}^{\perp}\left(-T_{1}\right)}{\operatorname{det} 2 \mathbf{\Omega}_{2}^{\perp}}=\operatorname{det} \tilde{\mathbf{J}}^{\perp}\left(-T_{1}\right),
$$

which demonstrates the equivalence of evaluating the Jacobi fields by starting the propagation from minimum 1 toward minimum 2 and vice versa. Exchanging columns of block matrices in Eq. (29) and multiplying the second row matrices by -1 , both of which introduce factors of $(-1)^{f-1}$, show that $\operatorname{det} \mathbf{Y}$ is symmetric with respect to 
changing the initial direction of the instanton trajectory (whether it is along the mode of frequency $\omega_{1}$ or $\omega_{2}$, as is necessary due to time reversal symmetry).

Finally, we equate Eqs. (29) and (30) evaluated at $t=0$ and insert the resulting expression for $\boldsymbol{\eta}\left(T_{2}\right)$ in Eq. (21) to obtain

$$
\operatorname{det} \mathbf{J}^{\perp}\left(T_{2}\right)=\operatorname{det}\left(\frac{\mathbf{A}^{\perp}(0)-\tilde{\mathbf{A}}^{\perp}(0)}{2}\right) \frac{\operatorname{det} \boldsymbol{\eta}(0) \operatorname{det} \tilde{\boldsymbol{\eta}}(0)}{\operatorname{det} 2 \boldsymbol{\Omega}_{1}^{\perp} \operatorname{det} \boldsymbol{\Omega}_{2}^{\perp}} .
$$

The significance of Eq. (32) is that it expresses the determinant of the perpendicular-mode Jacobi fields det $\mathbf{J}^{\perp}$, with initial conditions at $t=-T_{1}$, in the large time $T_{2}$ limit without propagating into the unstable region near the opposite-side minimum. $\mathbf{A}^{\perp}$ is propagated forward in time using the Riccati equation, Eq. (20), with initial conditions $\mathbf{A}^{\perp}\left(-T_{1}\right)=\mathbf{\Omega}_{1}^{\perp}$, toward minimum 2 until $t=0$. $\tilde{\mathbf{A}}^{\perp}$ is propagated backward in time starting at minimum 2 , with $\tilde{\mathbf{A}}^{\perp}\left(T_{2}\right)=-\boldsymbol{\Omega}_{2}^{\perp}$, toward minimum 1 until the meeting point with $\mathbf{A}^{\perp}$ at $t=0$. Determinants of $\boldsymbol{\eta}(0)$ and $\tilde{\boldsymbol{\eta}}(0)$ in Eq. (32) are obtained by integrating the trace of the log derivative of the Jacobi fields using Eq. (22).

We are now ready to collect all terms together to obtain the tunneling matrix elements. Equations (13), (18), and (32) are inserted into Eq. (11), which gives the tunneling splitting via Eq. (4). $P$ and $Q$ are substituted from Eq. (A4). The determinants of $\boldsymbol{\eta}(0)$ and $\tilde{\boldsymbol{\eta}}(0)$ are expressed using Eq. (22), with $\operatorname{det} \boldsymbol{\Omega}_{0}=\omega_{1} \operatorname{det} \boldsymbol{\Omega}_{1}^{\perp}=\omega_{2} \operatorname{det} \boldsymbol{\Omega}_{2}^{\perp}$ and $\operatorname{Tr} \mathbf{A}=d p / d x+\operatorname{Tr} \mathbf{A}^{\perp}$, as before. Taking the limits $T_{1,2} \rightarrow \infty$, we finally obtain

$$
\begin{aligned}
h_{12}= & -\frac{1}{\sqrt{\pi}} \mathrm{e}^{-S_{0}} \sqrt{\frac{\operatorname{det} \boldsymbol{\Omega}_{0}}{\operatorname{det}\left(\frac{\mathbf{A}^{\perp}(0)-\tilde{\mathbf{A}}^{\perp}(0)}{2}\right)}} p(0) \\
& \times \exp \left[\frac{1}{2} \int_{-\infty}^{0}\left(\operatorname{Tr} \boldsymbol{\Omega}_{0}-\operatorname{Tr} \mathbf{A}(t)\right) d t\right] \\
& \times \exp \left[\frac{1}{2} \int_{0}^{\infty}\left(\operatorname{Tr} \boldsymbol{\Omega}_{0}+\operatorname{Tr} \tilde{\mathbf{A}}(t)\right) d t\right],
\end{aligned}
$$

which is the main result of this paper. The tunneling matrix element connecting minima 1 and 2 is expressed in terms of convergent integrals and log derivatives of Jacobi fields at the connection point defined at $t=0$. The connection point is most conveniently taken as the maximum of the barrier along the MAP. The log derivatives of the two linearly independent solutions of the Jacobi equation $\mathbf{A}$ and $\tilde{\mathbf{A}}$ are obtained by integration of the Riccati equation from minima 1 and 2 inward until the connection point at $t=0$. The integration over imaginary time $t$ is conveniently performed along the instanton using the arc length distance coordinate, with $d t=d x / \sqrt{2 V(x)}$. Singularities at the start of the integrations are avoided as described in Appendix D. The determinant of the Jacobi field matrix in Eq. (33), projected onto the subspace perpendicular to the instanton, is obtained by multiplying the product of its eigenvalues by the factor $\sum_{i}\left|\mathbf{U}_{: i}^{\top} \boldsymbol{\tau}\right|^{2} \omega_{i}^{-1}$, where $\mathbf{U}_{: i}$ and $\omega_{i}$ are eigenvectors and eigenvalues of the Jacobi matrix, respectively, and $\boldsymbol{\tau}$ is the tangent vector to the instanton trajectory at $\mathbf{x}(0)$.

Before proceeding to numerical tests, we first check the consistency between the expressions for the tunneling matrix elements obtained above. For the purpose of comparison, we place the point $t=0$ in the harmonic vicinity of minimum 2 in both equations. The first integral in Eq. (33) is then equal to the integral in Eq. (24), whereas the second integral vanishes. Appendix A shows $Q=p(0)$ and the ratio of determinants in Eq. (33) is equal to $\omega_{2}$, the frequency of the mode that is parallel to the instanton at minimum 2 . We thus see that in this particular limit, the expressions for $h_{12}$ coincide.

\section{NUMERICAL TESTS}

In the numerical tests below, we calculate the tunneling splittings for malonaldehyde, and water dimer and trimer in order to test Eq. (33). All instanton paths were obtained using the string method ${ }^{10}$ with several hundred equally spaced beads in mass-scaled distances using Cartesian coordinates. The convergence criterion for the string optimization was set on the component of the action gradient perpendicular to the instanton as $\max \left|\nabla_{i} S^{\perp}\right|<10^{-7}$ a.u. for all beads $i$. These are overconverged values for the test calculations of the fluctuation prefactor. The instanton path was parameterized in terms of the mass-scaled arc length distance from minimum $1, x$, and interpolated in each Cartesian coordinate using natural cubic splines ${ }^{67}$ (and reinterpolated using more accurate arc lengths obtained from a previous spline interpolation). Vectors tangent to the instanton path are obtained as $\boldsymbol{\tau}=d \mathbf{x} / d x$. The potential curve along the MAP is interpolated by hermite interpolation using the potential and its derivatives at each bead. The derivatives, $d V / d x$, are obtained by projection of the potential gradients along the instanton, $\boldsymbol{\tau}$. Hessians are evaluated for every bead by finite differences of two gradients per bead (with $10^{-2} a_{0}$ displacements). Hessian matrices are "purified" by setting the eigenvalues for translations and rotations to zero explicitly. For this purpose, we construct the eigenvectors of translation and rotation, $\mathbf{u}_{k}$, at a bead $\mathbf{x}_{i}(x)$, as described in Appendix B of Ref. 50, and use them to build a projection matrix, $\mathbf{P}(x)=\mathbf{I}-\sum_{k} \mathbf{u}_{k} \mathbf{u}_{k}^{\top}$. The purified hessians, $\mathbf{P}(x) \mathbf{H}(x) \mathbf{P}(x)$, were interpolated element-by-element in terms of the arc length distance along the instanton path. Each time a hessian was invoked, the projection was applied again at the interpolated position $x$.

The Riccati equation, Eq. (20), was solved using a RungeKutta integration of fifth order (RK5). ${ }^{67}$ The difference between the RK5 and RK4 solutions, which was obtained using the same integration grid, was used for adjusting the accuracy via a stepsize control algorithm. ${ }^{67}$ The accuracy parameter atol was set to $10^{-12}$, rtol and $\epsilon$ to $10^{-6}$, in Eqs. (17.2.9) and (17.2.11) of Ref. 67 added together. These settings worked well in all cases. The matrices $\mathbf{A}(\epsilon)$ and $\tilde{\mathbf{A}}(\epsilon)$ at the start of the integration were obtained through the procedure described in Appendix D. Unless otherwise specified, corrections up to the second order are calculated using $\epsilon=0.1 m_{e}^{1 / 2} a_{0}$. Integrations in Eqs. (24) and (33) were performed in the arc length distance variable $x$, instead of imaginary time $t$, with the connection point set at the halfway distance between the two minima.

The theory for constructing the Jacobi field determinant from two independent solutions of the Riccati equation assumes that $\mathbf{A}$ are non-singular. We therefore exclude the eigenvectors having zero eigenvalues that belong to translations and rotations, from the treatment. This is accomplished by means of the projection matrix, which is applied as $\mathbf{P}(x) \mathbf{A}(x) \mathbf{P}(x)$ after every RK step throughout the integration. The modes of $\mathbf{A}$ do couple to rotations for some systems ${ }^{58}$ and this is where the RPI and Jacobi fields method will give different results. 


\section{A. Malonaldehyde}

The test calculations on malonaldehyde were performed on the analytic full-dimensional PES $(f=27)$ by Guo et al. ${ }^{11}$ The planar part of the potential was modified as in Ref. 43. Gradients were also obtained analytically. The transition state energy on this potential is $3497 \mathrm{~cm}^{-1}$, whereas on a realistic potential ${ }^{16}$ it is $1438 \mathrm{~cm}^{-1}$. The tunneling splitting obtained using the RPI method is reported ${ }^{47}$ to be $51 \mathrm{~cm}^{-1}$. Mil'nikov and Nakamura obtained $57.7 \mathrm{~cm}^{-1}$. We repeated the RPI calculations using $N=1200$ and $\beta=12000$ a.u. and obtained a splitting of $50.7 \mathrm{~cm}^{-1}$, and an action $S=8.11 \hbar$. In contrast, the string method optimization ${ }^{10}$ requires only $N=18$ to calculate the tunneling splitting within a $3 \%$ error margin. The experimental result is $21.6 \mathrm{~cm}^{-1}$. ${ }^{68}$ The potential profile along the instanton path is symmetric and is shown in Fig. 1. The barrier height along instanton is $4756 \mathrm{~cm}^{-1}$, with a clear corner-cutting effect. The path length in mass-scaled coordinates is $99.3 m_{e}^{1 / 2} a_{0}$, whereas a straight-line distance between the minima is $71.0 \mathrm{me}_{e}^{1 / 2} a_{0}$.

The propagation of $\mathbf{A}(x)$ using the Riccati equation from minimum 1 toward minimum 2 was carried out as described above and its eigenvalues along the path are shown in Fig. 2. The integration can be performed without numerical difficulties until $\approx 85 \%-90 \%$ of the total distance to minimum 2, depending on the accuracy used to converge the instanton path. At that point, when analyzed in the local coordinate frame obtained by the parallel transport of normal-mode axes from minimum 1, the mode along the instanton becomes unstable. The projection of $\mathbf{A}$ along the instanton, $A^{\|} \equiv \boldsymbol{\tau}^{\top} \mathbf{A} \boldsymbol{\tau}=d p / d x$, is a ratio of two small quantities $(\dot{p}$ and $p)$, in which numerical error accrues during the propagation. We have also checked other elements of $\mathbf{A}$, specifically those that couple the tangential mode to perpendicular modes in the local frame, as they can be expressed ${ }^{43}$ as products of the curvatures of the instanton path and the instanton speed, but found all of them to be stable until the close proximity of minimum 2 . In order to obtain a numerical value for the splitting from Eq. (24), we replace the tangential matrix element of $\mathbf{A}$ by $d p / d x$, calculated from the potential and its

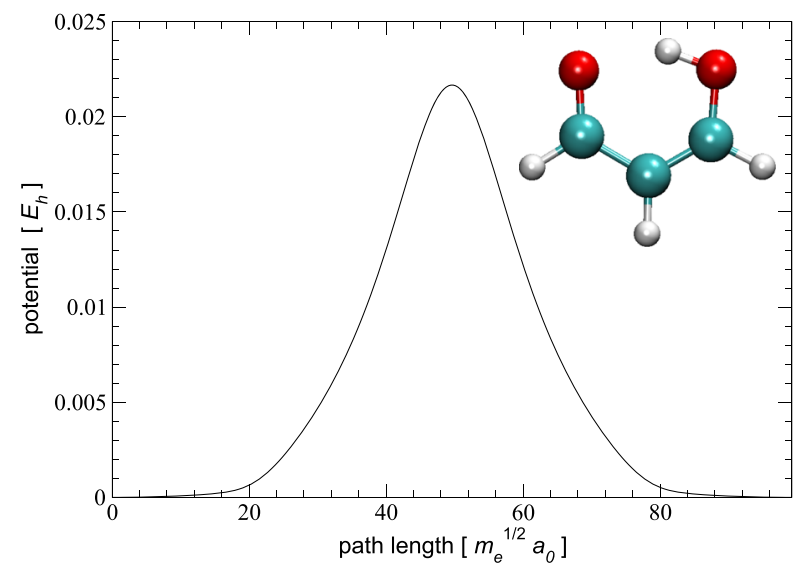

FIG. 1. Potential energy curve of the model potential of malonaldehyde ${ }^{11}$ along the instanton path in mass-scaled coordinates. The minimum-energy geometry is also shown.

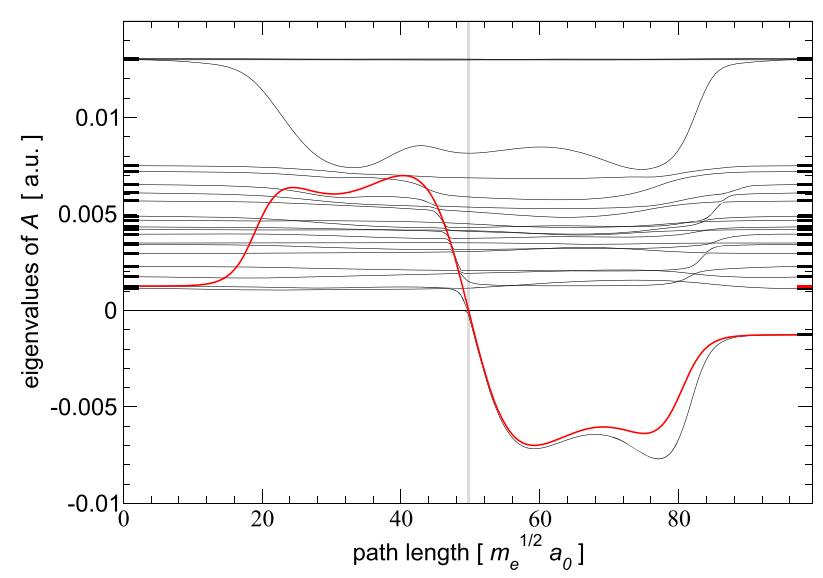

FIG. 2. Eigenvalues of the log derivative $A$ of Jacobi fields along the instanton path as a function of arc length distance from minimum 1. The red line is the projected value of $\mathbf{A}$ along the instanton path. It can be seen that the eigenvalues tend to frequencies of normal modes at minima, apart from the mode along which the instanton path approaches minimum 2, which changes sign, as indicated along the $y$ axis.

derivative $d V / d x$, as

$$
\mathbf{A}+\left(V^{\prime}(x) / \sqrt{2 V(x)}-\boldsymbol{\tau}^{\top} \mathbf{A} \boldsymbol{\tau}\right) \boldsymbol{\tau} \boldsymbol{\tau}^{\top} .
$$

In this way, the A propagation can be stabilized until $\approx 0.1 \mathrm{~m}^{1 / 2} a_{0}$ away from minimum 2, i.e., $99.9 \%$ of the way.

As shown in Fig. 2, the eigenvalues of $\mathbf{A}$ do not possess the mirror symmetry with respect to the center of the path, as the potential does. The instanton trajectory approaches both minima along the vibrational mode with the second-lowest frequency of $\omega_{\mathrm{ii}}=277 \mathrm{~cm}^{-1}$. Perpendicular eigenmodes of $\mathbf{A}$ coincide at both ends with vibrational frequencies of malonaldehyde. The parallel component $A^{\|}=d p / d x$, which is shown in Fig. 2 with a solid red line, is antisymmetric with respect to the center of the path. It starts from $\omega_{1}=\omega_{\mathrm{ii}}$ at minimum 1 and approaches $-\omega_{2}=-\omega_{\mathrm{ii}}$ near minimum 2. In general, it does not coincide with an eigenmode of $\mathbf{A}$ at intermediate positions.

The integrand in Eq. (24), $\left[\operatorname{Tr} \mathbf{\Omega}_{0}^{\prime}(0)-\operatorname{Tr} \mathbf{A}(x)\right] / \dot{x}$, is plotted against the path length $x$ using a solid black line in Fig. 3 . The area under the curve is the value of the integral in Eq. (24). The jump at the midpoint of the path reflects the change of sign of $\omega_{2}$ in the sum of frequencies $\operatorname{Tr} \Omega_{0}^{\prime}$. The dotted line in Fig. 3 shows the exponential divergence of the integrand, as $\exp \left(2 \omega_{2} t\right)$, had we not flipped the sign of $\omega_{2}$. From the position of the jump at $x(t=0)$ onward, the integration of $Q$ using Eq. (A5) is performed. The integrand is shown in Fig. 3 using a solid red line, and the area under the curve is the value of the exponent in Eq. (A5). The $Q$ integration also becomes unstable when approaching minimum 2 , due to the division by the instanton speed. We thus stop the integration when the integrand becomes small and obtain $Q=3.51 \times 10^{-2}$ a.u. We obtained the tunneling splitting of $50.8 \mathrm{~cm}^{-1}$, which is in excellent agreement with the RPI value.

We also obtained the splitting of $50.8 \mathrm{~cm}^{-1}$ using Eq. (33). For a symmetric path, the two integrals in Eq. (33) are equivalent. The integration from minimum 2 in negative imaginary time 


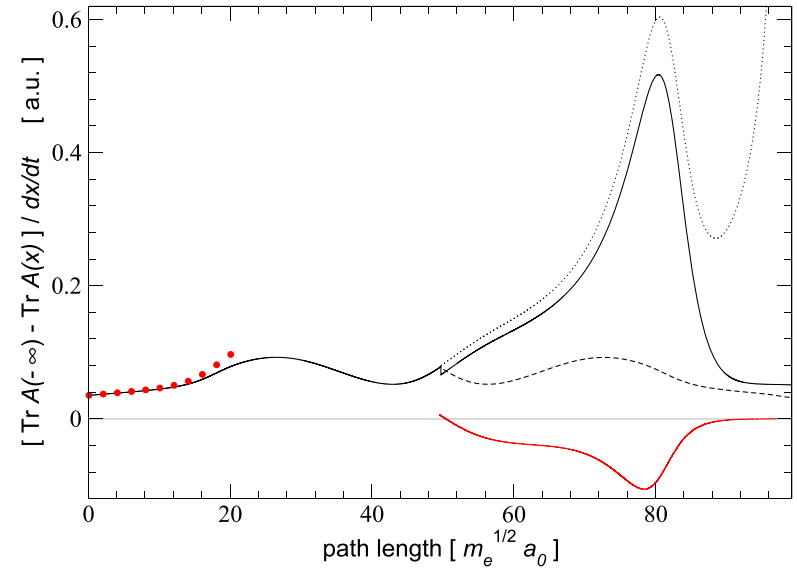

FIG. 3. Integrand of Eq. (24) divided by the instanton speed as a function of arc length distance from minimum 1 along the instanton path is shown using a solid black curve. The dotted curve shows the divergence of the integral if the frequency of $\omega_{2}$ does not change sign at $t=0$ (see text for details). The integrand of Eq. (3 is shown using a dashed line. The red line shows the integrand in Eq. (A5), needed to obtain $Q$ in Eq. (24). The integrand obtained using the expansion in Eq. (D9) is shown using red dots.

steps can be converted into the integration over $x$, with the integration limits swapped, and $\operatorname{Tr} \tilde{\mathbf{A}}=-\operatorname{Tr} \mathbf{A}$. The dashed line in Fig. 3 shows the second integrand (divided by $\dot{x}$ ) in Eq. (33), which is a mirror image of the first integrand. Nevertheless, at the connection point $-\tilde{\mathbf{A}} \neq \mathbf{A}$, and $(\mathbf{A}-\tilde{\mathbf{A}}) / 2$ in Eq. (33) cannot be replaced by $\mathbf{A}$, because hessians at minimum 1 and minimum 2 (which are used to set the starting values of $\mathbf{A}$ and $\tilde{\mathbf{A}}$ ) are related by a permutation-inversion operation, and their indices refer to different atom coordinates for the two versions. The permutation-inversion operation, however, does not affect the trace in the integral. The formula for the tunneling splitting, Eq. (100) in Ref. 43, therefore does not apply even for a symmetric system and, in our calculation, gives $57.3 \mathrm{~cm}^{-1}$.

We have also checked that the tunneling splittings obtained using Eq. (33) do not depend on the position of the connection point. This proved to be valid everywhere, apart from the close vicinity of minima, provided that translations and rotations are projected out from $\mathbf{A}$ at every RK integration step, as described above. The matching results of the RPI and JFI methods thus prove that it is justifiable to use the Jacobi fields method in Cartesian coordinates ${ }^{43}$ and that for a system, such as malonaldehyde, where coupling to rotations does not significantly affect the result, the two theories give the same results.

The red dotted line in Fig. 3 shows the integrand in Eq. (33) calculated using the expansion up to quadratic terms in $x$, as described in Appendix D, which is used to set the value of $\mathbf{A}$ at the start of the integration. The exceptional accuracy of the expansion away from minima is probably an artifact of a large harmonic region around the minima and the absence of rotations at the start of the instanton path in the model potential.

We proceed below to test the theory on a realistic potential and on asymmetric paths.

\section{B. Water dimer and trimer}

There are several state-of-the-art water potentials, which all use a many-body expansion with flexible monomers. ${ }^{69-71}$ We use the Wang-Huang-Braams-Bowman (WHBB) potential ${ }^{71,72}$ for our tests. Instanton calculations of tunneling matrix elements on the WHBB potential and the analysis of the splitting pattern in terms of rearrangements have been reported in Ref. 30 .

The minimum geometry of the water dimer is shown labeled in Fig. 4. There are eight degenerate wells, related by permutations of atoms, between which the system can tunnel without breaking monomer bonds over five different rearrangement paths. The rearrangement paths are listed in Table I with their associated permutation operation. The potential profile along the MAP for each rearrangement is shown in Fig. 4. Four of them are symmetric, while the donor exchange (12) is asymmetric.

The tunneling matrix elements were calculated using the RPI and JFI methods with Eq. (33) and are given in Table I. The splitting pattern can be obtained analytically in terms of the matrix elements. ${ }^{30}$ The action converges easily and is the same for both methods. RPI results are obtained with $\beta=30.000$ a.u. and $N=800$. The last digit shown in the RPI matrix elements is not reliable due to a slow convergence of the method. ${ }^{50}$ The RPI matrix elements, which vary over several orders of magnitude, differ by up to almost $38 \%$ from the JFI results.

We analyzed the asymmetric donor-exchange path in more detail. The relative discrepancy between the RPI and JFI results is the largest for this case. The low-lying eigenvalues of the hessian (red lines), A, and $\tilde{\mathbf{A}}$ (black lines) are shown in Fig. 5. The connection point is in the center of the path and the asymmetry is apparent. The low-lying vibrational modes significantly mix with external modes. We note that the explicit projection of rotations and translations from the hessians significantly affects the RPI results (independent of the step size in the finite difference formula for the calculation of hessians), by $80 \%$ for the donor exchange. If coupling of $\mathbf{A}$ to rotations is not projected out, the eigenmodes of $\mathbf{A}$ are also significantly affected, as shown by the gray lines in Fig. 5. Due to the numerical

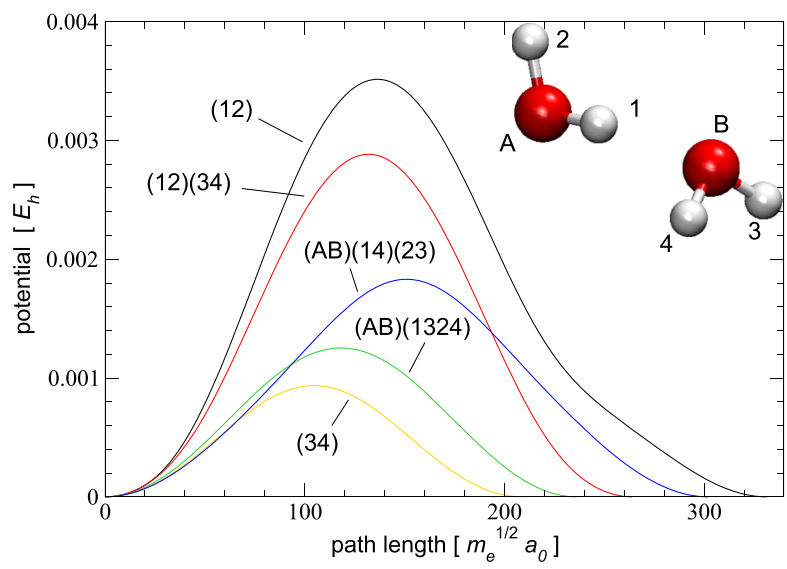

FIG. 4. Potential energy curves of the water dimer along the various instanton tunneling paths as labeled by the permutation operations that connect the minima joined by the pathways. The minimum-energy geometry labeled in the reference version is also shown. 
TABLE I. Tunneling splitting matrix elements and actions for the water dimer on the WHBB potential ${ }^{71,72}$ using JFI. Exponents are given in parentheses. The RPI results using $\beta=30.000$ a.u. and $N=800$ are included for comparison.

\begin{tabular}{lcccc}
\hline \hline $\begin{array}{l}\text { Path } \\
\text { name }\end{array}$ & Permutation & $\begin{array}{c}\text { JFI } \\
\left(\mathrm{cm}^{-1}\right)\end{array}$ & Action/ $\hbar$ & $\begin{array}{c}\text { RPI } \\
h\left(\mathrm{~cm}^{-1}\right)\end{array}$ \\
\hline $\begin{array}{l}\text { Accept. tunnel. } \\
\text { Geared interch. }\end{array}$ & $(\mathrm{AB})(1324)$ & 0.17 & 7.63 & 4.6 \\
Bifurcation & $(12)(34)$ & $2.5(-2)$ & 12.00 & 0.15 \\
Anti-gear. int. & $(\mathrm{AB})(14)(23)$ & $2.9(-2)$ & 11.16 & $2.1(-2)$ \\
Donor exchange & $(12)$ & $1.8(-3)$ & 15.91 & $1.3(-3)$ \\
\hline
\end{tabular}

error in hessians and the mixing of rotations, which the JFI and RPI methods treat differently, the scale of the disagreement between the JFI and RPI results is within the expected limits. It should be noted that previous RPI results ${ }^{30}$ also differ from those reported here due to differences in the PES, as the paths have different actions, despite the actions converging rapidly.

In order to further test the result for an asymmetric path, we calculated the matrix element for the donor exchange path in the water dimer using Eq. (24). The left minimum is approached along the vibrational mode with the lowest frequency $\omega_{\mathrm{i}}=128 \mathrm{~cm}^{-1}$, whereas minimum 2 along $\omega_{\mathrm{iii}}=150 \mathrm{~cm}^{-1}$. In Fig. 6, the integrand in Eq. (24) is shown with a dotted line, and the integrands in Eq. (33) with solid lines. The integration of $\mathbf{A}$ in Eq. (24) is stable to $\approx 80 \%$ of the total arc length distance between minima. Using Eq. (34), we can propagate up to $99 \%$ of the full distance. We obtain the splitting of $1.8 \times 10^{-3} \mathrm{~cm}^{-1}$ in excellent agreement with the result obtained using Eq. (33). The integral in Eq. (24) was extrapolated using the last integrand multiplied by the remaining distance to minimum 2. This remainder accounts for $\approx 4 \%$ of the matrix element $h$ and an uncertainty of that magnitude is therefore expected in the final result.

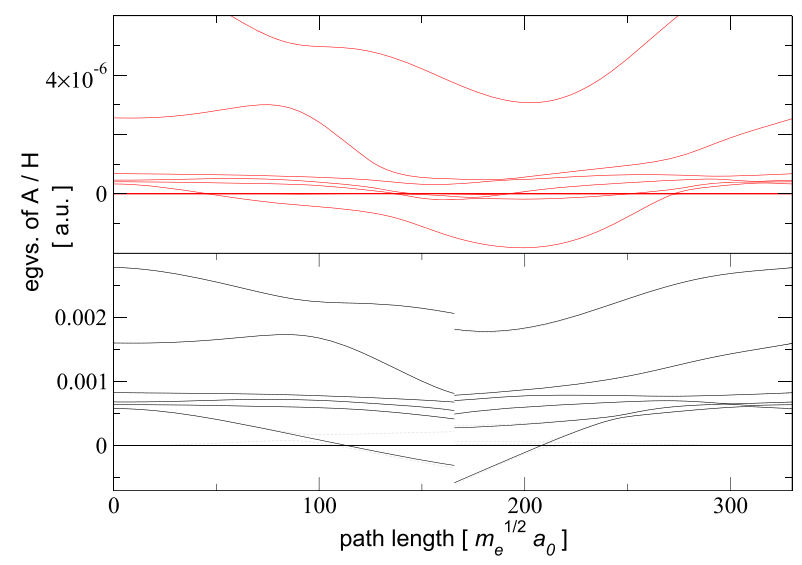

FIG. 5. Eigenvalues of the hessian and of the log derivative $\mathbf{A}$ of Jacobi fields along the water dimer (12) instanton path as a function of arc length distance from minimum 1 are shown in the upper and lower panels with red and black solid lines, respectively. Dashed gray lines in the lower panel show the non-zero coupling of the modes of $\mathbf{A}$ to the external rotations.

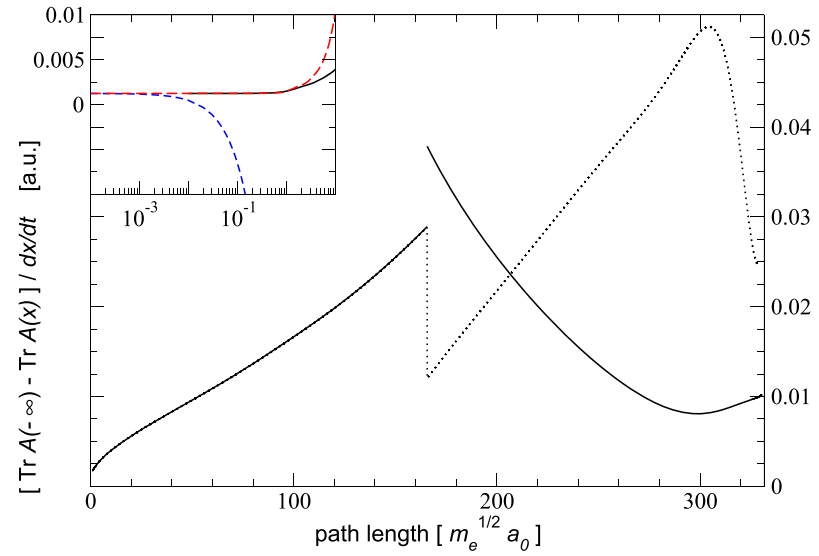

FIG. 6. Integrands of Eqs. (24) and (33) divided by the instanton speed as a function of arc length distance from minimum 1 along the instanton path are shown using dotted and solid black curves, respectively. The integrand obtained using the expansion in Eq. (D9) up to linear (dashed blue) and quadratic (dashed red line) terms is shown in the inset.

We mention here that the result obtained using Eq. (33) does not depend on the connection point. If we do not project out the rotations from $\mathbf{A}$, however, the results vary depending on the position of the connection point by about as much as the differences discussed above due to different treatments of rotational modes.

The inset in Fig. 6 shows the behavior of the integrand near minimum 1, calculated using a linear (dashed blue) and a quadratic (dashed red) expansion of $\mathbf{A}$ in $x$. The adaptive step size RK5 method takes a large number of steps near the minima as its stability degrades. A large first step to $x=\epsilon$ is therefore advantageous. With a linear correction in $\mathbf{A}$, we obtain the correct limit, but RK5 does not guarantee stability to such small distances $\left(<10^{-3} m_{e}^{1 / 2} a_{0}\right)$. The considerable improvement brought by the quadratic correction $x$ is therefore desirable to take the RK5 integration away from singularities at the minima.

We also calculated the tunneling matrix elements in the water trimer. The water trimer minimum geometry, labeled in its reference version, is shown in Fig. 7. The minimum structure is a ring where each water monomer acts as a hydrogen bond donor and acceptor. The 96 minima accessible via tunneling motions are responsible for the splitting pattern, which can be explained in terms of six motions. ${ }^{30}$ The most labile motion is the flip of hydrogen 2 from above the ring plane formed by the oxygen atoms, to below the plane. Another contributing motion is a bifurcation, where hydrogen 1 rotates out of the bond below the ring plane, and hydrogen 2 moves into the bond, from above the ring plane. The remaining motions are the combinations of bifurcations accompanied by one or two simultaneous flips. The potential profiles of the rearrangements along the instanton pathways are shown in Fig. 7. Paths are labeled by the symbols of oxygen atoms of monomers involved in the flips of the hydrogen bonds and, if capped by a tilde, in the bifurcation dynamics of the hydrogen bonds. The labels used in Ref. 30 are defined in Table II. Paths B1 and B3 are symmetric, A1 and $\mathrm{A} 3$ are nearly symmetric, and the flip and $\mathrm{A} 3$ are asymmetric. 


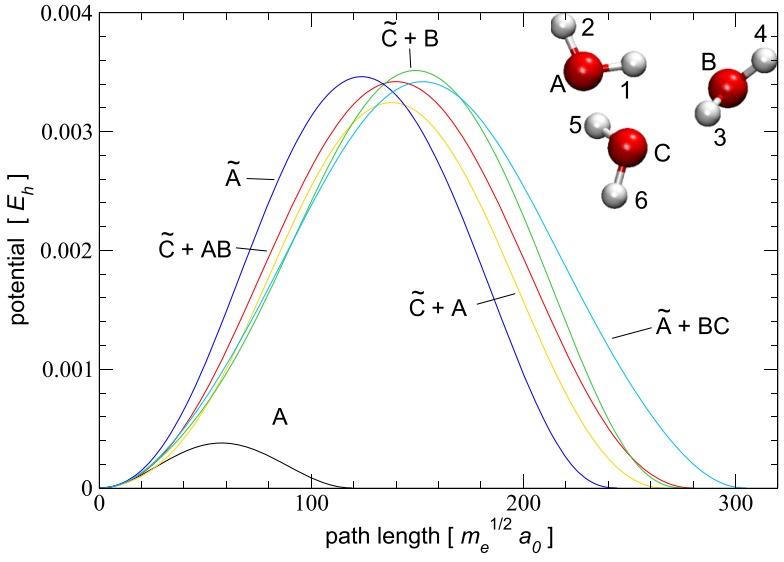

FIG. 7. Potential energy curves of the water trimer along the various instanton tunneling paths as labeled. The minimum-energy geometry labeled in the reference version is also shown. Paths are labeled by the symbols of oxygen atoms of monomers involved in the flip of the hydrogen bond and, if capped by a tilde, in the bifurcation dynamics of the hydrogen bond.

For asymmetric paths, the motion in different directions is labeled differently in terms of atom labels, as atoms take on different roles in the minima they connect. Both labels are given in Table II.

The tunneling matrix elements obtained using JFI and RPI are listed in Table II. The RPI results are obtained with $\beta=20.000$ a.u. and $N=600(N=800$ for the flip). The last digit shown in the RPI matrix elements is again unreliable. Nevertheless, the agreement is excellent and within the error bars. The purification of hessians does not affect the results significantly, and rotations do not couple appreciably to the instanton motion, so JFI and RPI give the same results to within the numerical error bars. The newer version of the WHBB potential treats 3-body terms differently in the potential, so the results differ somewhat from Ref. 30 . The magnitude of these differences can be explained entirely by the differences in the actions (given in Table II).

The above results thus demonstrate that the tunneling matrix elements can be obtained using the JFI method with Eq. (33) in Cartesian coordinates. It is necessary to decouple translations and rotations from the treatment and, for systems where this is a valid

TABLE II. Tunneling splitting matrix elements and actions for the water trimer on the WHBB potential ${ }^{71,72}$ using JFI. Exponents are given in parentheses. The RPI results obtained using $\beta=20.000$ a.u. and $N=600$ ( $N=800$ for the flip) are included for comparison.

\begin{tabular}{lcccc}
\hline \hline $\begin{array}{l}\text { Path } \\
\text { symbol }\end{array}$ & Mechanics & $\begin{array}{c}\text { JFI } \\
h\left(\mathrm{~cm}^{-1}\right)\end{array}$ & Action/ $/ \hbar$ & $\begin{array}{c}\text { RPI } \\
h\left(\mathrm{~cm}^{-1}\right)\end{array}$ \\
\hline Flip & $\mathrm{A} / \mathrm{B}$ & 48.5 & 2.11 & 48.4 \\
$\mathrm{~A} 1$ & $\tilde{\mathrm{C}}+\mathrm{A} / \tilde{\mathrm{B}}+\mathrm{C}$ & $5.9(-3)$ & 12.96 & $5.9(-3)$ \\
$\mathrm{A} 2$ & $\tilde{\mathrm{C}}+\mathrm{B} / \tilde{\mathrm{A}}+\mathrm{C}$ & $1.4(-2)$ & 14.18 & $1.4(-2)$ \\
$\mathrm{A} 3$ & $\tilde{\mathrm{A}} / \tilde{\mathrm{B}}$ & $7.0(-3)$ & 12.55 & $7.0(-3)$ \\
$\mathrm{B} 1$ & $\tilde{\mathrm{C}}+\mathrm{AB}$ & $1.5(-2)$ & 14.16 & $1.6(-2)$ \\
$\mathrm{B} 3$ & $\tilde{\mathrm{A}}+\mathrm{BC}$ & $1.3(-2)$ & 15.64 & $1.3(-2)$ \\
\hline \hline
\end{tabular}

approximation, the agreement with RPI was established. In systems where rotation couples significantly to the instanton trajectory, such as the donor exchange in water dimer, the newly derived Eq. (33) gives results independent of the connection point and in agreement with Eq. (24).

\section{CONCLUSIONS}

We have derived the JFI method for calculating tunneling matrix elements for general paths, which is a generalization of the Mil'nikov-Nakamura theory in Ref. 43 to asymmetric paths. Once the MAPs have been determined, the calculation of tunneling matrix elements connecting any two degenerate wells reduces to propagating stable solutions of the Riccati equation from each well toward each other until a connection point is reached. An additional approximation is the neglect of all dynamical couplings to external rotation, which serves to make the formula independent of the position of the connection point.

The MAP shape is determined numerically by an optimization using either the string method with LBFGS or the quadratic string method. The optimized path shape is defined by $N$ equally spaced discretization points, or beads, without reference to the imaginary time period $\beta$. In contrast to the RPI method, the calculation of the fluctuation prefactor can also be performed without reference to the imaginary time, by integration of the Riccati equation along the MAP. In practice, this means that the optimization of the path and the calculation of the fluctuation prefactor are performed fewer times. The convergence with the imaginary time period is effectively accomplished automatically through the adaptive variation of the step size in the integration of the Riccati equation. Once the MAP is found, the final calculation of the splittings is done in a negligible amount of time using Eq. (33), avoiding the expensive diagonalizations of RPI. The present development therefore simplifies the process and practically turns the calculation of tunneling matrix elements into a black-box method that is valid for general Cartesian paths.

The JFI method was tested on a model potential of malonaldehyde, the water dimer, and the water trimer by comparing the results to the RPI method. We have also tested the formula against a tailored one-way propagation using Eq. (24). All results are in good agreement, with the present approach being significantly more efficient.

Based on the work of Ref. 73, the JFI method can be extended to the calculation of tunneling splittings in vibrationally excited states. The Jacobi field method also requires fewer potential evaluations than the RPI method, as it relies on the string method, and is therefore especially suited for application to large systems, systems that exhibit slow motion of the heavy-atom skeleton, or in combination with expensive on-the-fly potentials. The calculation of tunneling splittings using our method is as numerically intensive as locating the minimum energy paths, making the range of possible applications very broad.

\section{ACKNOWLEDGMENTS}

This work was supported by Croatian Science Foundation Grant Nos. IP-2014-09-7540 and IP-2016-06-1142, and in part by the QuantiXLie Centre of Excellence, a project cofinanced by the 
Croatian Government and European Union through the European Regional Development Fund - the Competitiveness and Cohesion Operational Program (Grant No. KK.01.1.1.01.0004).

\section{APPENDIX A: CALCULATION OF $P$ AND $Q$}

The double-well potential is assumed to be harmonic near the minima. The classical zero-energy trajectory $x(t)$ connects minima 1 and 2 on the inverted potential. The trajectory starts from minimum 1, moving along the normal mode having frequency $\omega_{1}$, and approaches minimum 2 along the normal mode $\omega_{2}$. The origin of the imaginary time $t$ is located between the two minima at an arbitrary geometry. The trajectory $x(t)$ approaches the minima at large times $t=-T_{1}$ and $T_{2}$ with an exponentially decaying speed (or momentum $p$ in mass-scaled coordinates),

$$
\begin{aligned}
p\left(-T_{1}\right) & =P \mathrm{e}^{-\omega_{1} T_{1}}, \\
p\left(T_{2}\right) & =Q \mathrm{e}^{-\omega_{2} T_{2}} .
\end{aligned}
$$

Using the chain rule, one obtains the relation

$$
\int_{A}^{B} d \ln p(x(t))=\int_{A}^{B} \frac{\partial p}{\partial x} d t
$$

which can be used to relate the momenta at times $A$ and $B$,

$$
p(B)=p(A) \exp \left[\int_{A}^{B} \frac{\partial p}{\partial x} d t\right] .
$$

The proportionality constants $P$ and $Q$ can then be found using Eqs. (A1) and (A3), giving

$$
\begin{aligned}
& P=p(0) \exp \left[\int_{-T_{1}}^{0}\left(\omega_{1}-\frac{\partial p}{\partial x}\right) d t\right], \\
& Q=p(0) \exp \left[\int_{0}^{T_{2}}\left(\omega_{2}+\frac{\partial p}{\partial x}\right) d t\right] .
\end{aligned}
$$

Here, $p(0)$ is the momentum at $x(0)$ and is easily obtained from the potential with $p(x) \equiv \dot{x}=\sqrt{2 V(x)}$. The integrals in Eq. (A4) converge because $d p / d x=\omega$ for a harmonic potential having frequency $\omega$. For two harmonic potentials joined at $x(0)$, we have $P=Q=p(0)$. The numerical evaluation of these (using $Q$ as an example) from the potential is achieved using

$$
Q=\sqrt{2 V(0)} \exp \left[\int_{x(0)}^{x\left(T_{2}\right)}\left(\omega_{2}+\frac{V^{\prime}(x)}{\sqrt{2 V(x)}}\right) \frac{d x}{\sqrt{2 V(x)}}\right],
$$

where the integration is propagated until $x\left(T_{2}\right)$ is such that the term inside the parentheses becomes negligible upon entering the harmonic region near the minimum.

\section{APPENDIX B: DERIVATION OF $\lambda$}

This derivation is based on Refs. 48 and 49; we follow the notation of Ref. 43. The lowest energy eigenvalue $\lambda$ of

$$
-\frac{d}{d t^{2}} \psi+V^{\prime \prime}(x(t))=\lambda \psi
$$

with boundary conditions $\psi\left(-T_{1}\right)=\psi\left(T_{2}\right)=0$ at large but finite $T_{1,2}$ can be obtained ${ }^{43,49}$ from the integral equation

$$
\psi(t)=\psi_{0}(t)+\lambda \int_{-T_{1}}^{t} d t^{\prime}\left[\eta(t) \xi\left(t^{\prime}\right)-\eta\left(t^{\prime}\right) \xi(t)\right] \psi\left(t^{\prime}\right),
$$

where $\psi_{0}$ is the solution for $\lambda=0$. The terms inside the brackets correspond to the Green's function constructed from $\eta$. When $T_{1}$ and $T_{2}$ are finite, the requirement $\psi_{0}\left(-T_{1}\right)=0$ adds a component of the exponentially growing solution of Eq. (B1) with $\lambda=0$, namely $\xi$, as

$$
\psi_{0}(t)=\eta(t)+C \xi(t) .
$$

The constant $C=2 \omega_{1} P^{2} \exp \left(-2 \omega_{1} T_{1}\right)$ is obtained by inserting the asymptotic behavior of $\eta$ and $\xi$ given by Eqs. (14) and (15) in Eq. (B3), and requiring $\psi\left(-T_{1}\right)=0$. One then inserts $\psi_{0}$ from Eq. (B3) into the right-hand side of Eq. (B2), obtaining the approximate expression for $\lambda$ [with the requirement that $\psi\left(T_{2}\right)=0$ in Eq. (B2)] as

$$
\begin{aligned}
\lambda & =\frac{\psi_{0}\left(T_{2}\right)}{\int_{-T_{1}}^{T_{2}} d t^{\prime}\left[\eta\left(T_{2}\right) \xi\left(t^{\prime}\right)-\eta\left(t^{\prime}\right) \xi\left(T_{2}\right)\right] \psi_{0}\left(t^{\prime}\right)} \\
& =\frac{\psi_{0}\left(T_{2}\right)}{\xi\left(T_{2}\right) \int_{-T_{1}}^{T_{2}} d t^{\prime} \eta^{2}\left(t^{\prime}\right)},
\end{aligned}
$$

where we have neglected exponentially small terms. As $\eta=\dot{x}$ is the instanton velocity, the integral in Eq. (B4) is recognized as the action $S_{0}$. Applying the asymptotic expressions for $\eta$ and $\xi$ in Eqs. (14) and (15) to Eqs. (B3) and (B4), we obtain

$$
\lambda=\left[2 \omega_{1} P^{2} \mathrm{e}^{-2 \omega_{1} T_{1}}+2 \omega_{2} Q^{2} \mathrm{e}^{-2 \omega_{2} T_{2}}\right] \frac{1}{S_{0}} .
$$

\section{APPENDIX C: USEFUL RELATIONS}

In this appendix, we list useful relations involving determinants. For matrices $\mathbf{A}, \mathbf{B}, \mathbf{C}$, and $\mathbf{D}$, the following relations are valid:

$$
\operatorname{det} \mathrm{e}^{\mathbf{A}}=\mathrm{e}^{\operatorname{Tr} \mathbf{A}} \text {, }
$$

$$
\left|\begin{array}{l}
\mathbf{A B} \\
\mathbf{C D}
\end{array}\right|=\operatorname{det} \mathbf{A} \operatorname{det}\left(\mathbf{D}-\mathbf{C A}^{-1} \mathbf{B}\right)=\operatorname{det} \mathbf{D} \operatorname{det}\left(\mathbf{A}-\mathbf{B D}^{-1} \mathbf{C}\right) .
$$

If $\mathbf{A}=\dot{\boldsymbol{\eta}} \boldsymbol{\eta}^{-1}$, then

and

$$
\operatorname{det} \dot{\boldsymbol{\eta}}=\operatorname{det} \mathbf{A} \operatorname{det} \boldsymbol{\eta}
$$

$$
\frac{d}{d t} \operatorname{det} \boldsymbol{\eta}=\operatorname{det} \boldsymbol{\eta} \operatorname{Tr} \mathbf{A} \text {. }
$$

\section{APPENDIX D: AVOIDING SINGULARITIES AT THE MINIMA}

The instanton trajectory spends an infinite amount of time near the minima, so the integration of the Riccati equation in Eq. (20) has to be started from a position $x=\epsilon$ that is slightly displaced from the minimum to avoid the singularity. In order to calculate the starting matrix $\mathbf{A}(\epsilon)$, we expand the matrix elements of the hessian $\mathbf{H}$, the log derivative of the Jacobi fields $\mathbf{A}$, and the instanton speed to quadratic terms in $x$,

$$
\begin{aligned}
& \mathbf{H}(x)=\mathbf{H}_{0}+x \mathbf{H}_{1}+x^{2} \mathbf{H}_{2}, \\
& \mathbf{A}(x)=\mathbf{A}_{0}+x \mathbf{A}_{1}+x^{2} \mathbf{A}_{2},
\end{aligned}
$$




$$
\dot{x}=\alpha x+\beta x^{2} .
$$

Here $\mathbf{A}_{0}=\boldsymbol{\Omega}_{0}$, such that $\mathbf{H}_{0}=\mathbf{A}_{0}^{2}$ and $\alpha=\omega_{1}$ for a purely quadratic potential with frequency $\omega_{1}$. In Eq. (D1), $\mathbf{H}_{1}$ and $\mathbf{H}_{2}$ are determined from hessians evaluated at $x=\epsilon / 2$ and $x=\epsilon$, whereas $\alpha$ and $\beta$ are determined from the potential values $V(\epsilon / 2)$ and $V(\epsilon)$. Equations (D1)-(D3) are then inserted into the Riccati equation,

$$
\dot{x} \frac{d \mathbf{A}(x)}{d x}=\mathbf{H}(x)-\mathbf{A}^{2}(x),
$$

and, by equating terms of order $\epsilon$ and $\epsilon^{2}$, we obtain

$$
\begin{gathered}
\alpha \mathbf{A}_{1}+\mathbf{A}_{0} \mathbf{A}_{1}+\mathbf{A}_{1} \mathbf{A}_{0}=\mathbf{H}_{1}, \\
2 \alpha \mathbf{A}_{2}+\mathbf{A}_{0} \mathbf{A}_{2}+\mathbf{A}_{2} \mathbf{A}_{0}=\mathbf{H}_{2}-\mathbf{A}_{1}^{2}-\beta \mathbf{A}_{1},
\end{gathered}
$$

respectively. These equations are solved by multiplying through with $\mathbf{U}^{\top}$ from the left and $\mathbf{U}$ from the right, where $\mathbf{U}$ is such that $\overline{\mathbf{A}}_{0}=\mathbf{U}^{\top} \mathbf{A}_{0} \mathbf{U}$ is diagonal. Equation (D5) yields

$$
\left[\overline{\mathbf{A}}_{1}\right]_{i j}=\frac{\left[\overline{\mathbf{H}}_{1}\right]_{i j}}{\alpha+\left[\overline{\mathbf{A}}_{0}\right]_{i i}+\left[\overline{\mathbf{A}}_{0}\right]_{j j}},
$$

where matrices capped by a bar are in the basis in which $\mathbf{A}_{0}$ and $\mathbf{H}_{0}$ are diagonal. Equation (D7) determines $\overline{\mathbf{A}}_{1}$ in terms of $\mathbf{H}_{1}$ and $\alpha$, which is used in turn with Eq. (D6) to obtain $\overline{\mathbf{A}}_{2}$ as

$$
\left[\overline{\mathbf{A}}_{2}\right]_{i j}=\frac{\left[\overline{\mathbf{H}}_{2}-\overline{\mathbf{A}}_{1}^{2}-\beta \overline{\mathbf{A}}_{1}\right]_{i j}}{2 \alpha+\left[\overline{\mathbf{A}}_{0}\right]_{i i}+\left[\overline{\mathbf{A}}_{0}\right]_{j j}} .
$$

$\mathbf{A}_{1}$ and $\mathbf{A}_{2}$ in Eq. (D2) are obtained by transforming $\overline{\mathbf{A}}_{1,2}$ back to the original basis using $\mathbf{U}$.

$\mathbf{A}(\epsilon)$ is used as a starting value at $x=\epsilon$ for the propagation of the Riccati equation in $x$ using the RK5 algorithm. ${ }^{67}$ The trace integral in Eqs. (24) and (33) along the instanton in the interval $x \in[0, \epsilon]$ is evaluated as

$$
\begin{aligned}
\int_{-\infty}^{t(\epsilon)} \operatorname{Tr}\left[\mathbf{A}_{0}-\mathbf{A}(x(t))\right] d t & =\int_{0}^{\epsilon} \frac{\operatorname{Tr}\left(-x \mathbf{A}_{1}-x^{2} \mathbf{A}_{2}\right)}{\alpha x+\beta x^{2}} d x \\
& \approx-\frac{\operatorname{Tr} \mathbf{A}_{1}}{\alpha} \epsilon-\frac{\operatorname{Tr} \mathbf{A}_{2}}{2 \alpha} \epsilon^{2}+\frac{\beta \operatorname{Tr} \mathbf{A}_{1}}{2 \alpha^{2}} \epsilon^{2} .
\end{aligned}
$$

The integral of the rational function in Eq. (D9) is analytic, but is easily evaluated as above for small $\beta$. We now observe that for a purely quadratic potential in the interval $[0, \epsilon], \mathbf{H}_{1}$ is zero, and so are $\mathbf{A}_{1}$ and the trace integral in Eq. (D9). If only linear terms are kept in the expansions of $\mathbf{H}(x)$ and $\mathbf{A}(x)$ in Eqs. (D1) and (D2), only the first term on the right-hand side of Eq. (D9) survives. The second term results from the $\epsilon^{2}$ term in $\mathbf{A}(x)$ expansion, whereas the third term results from the $\epsilon^{2}$ term in the instanton speed, Eq. (D3). In all cases encountered so far, we have found the third term to be negligible.

\section{REFERENCES}

${ }^{1}$ V. A. Benderskii, D. E. Makarov, and C. A. Wight, "Chemical dynamics at low temperatures," in Advances in Chemical Physics (Wiley, New York, 1994), Vol. 88. ${ }^{2}$ M. T. Cvitaš and S. C. Althorpe, Phys. Scr. 80, 048115 (2009).

${ }^{3}$ M. Schröder, F. Gatti, and H.-D. Meyer, J. Chem. Phys. 134, 234307 (2011).

${ }^{4}$ T. Hammer and U. Manthe, J. Chem. Phys. 134, 224305 (2011).

${ }^{5}$ H.-G. Yu, H. Song, and M. Yang, J. Chem. Phys. 146, 224307 (2017).
${ }^{6}$ R. Marquardt, K. Sagui, J. Zheng, W. Thiel, D. Luckhaus, S. Yurchenko, F. Mariotti, and M. Quack, J. Phys. Chem. A 117, 7502 (2013).

${ }^{7}$ X. Huang, S. Carter, and J. Bowman, J. Chem. Phys. 118, 5431 (2003).

${ }^{8}$ T. Kawatsu and S. Miura, Chem. Phys. Lett. 634, 146 (2015).

${ }^{9}$ A. R. Sharma, B. J. Braams, S. Carter, B. C. Shepler, and J. M. Bowman, J. Chem. Phys. 130, 174301 (2009).

${ }^{10}$ M. T. Cvitaš and S. C. Althorpe, J. Chem. Theory Comput. 12, 787 (2016).

${ }^{11}$ Y. Guo, T. D. Sewell, and D. L. Thompson, Chem. Phys. Lett. 224, 470 (1994)

${ }^{12}$ T. Carrington and W. H. Miller, J. Chem. Phys. 84, 4364 (1986).

${ }^{13}$ G. V. Mil'nikov, T. Ishida, and H. Nakamura, J. Phys. Chem. A 110, 5430 (2006).

${ }^{14}$ E. Kamarchik, Y. Wang, and J. Bowman, J. Phys. Chem. A 113, 7556 (2009).

${ }^{15}$ D. J. Nesbitt and F. Dong, Phys. Chem. Chem. Phys. 10, 2113 (2008).

${ }^{16}$ Y. Wang, B. J. Braams, J. M. Bowman, S. Carter, and D. P. Tew, J. Chem. Phys. 128, 224314 (2008).

${ }^{17}$ M. D. Coutinho-Neto, A. Viel, and U. Manthe, J. Chem. Phys. 121, 9207 (2004).

${ }^{18}$ C. S. Tautermann, A. F. Voegele, T. Loerting, and K. R. Liedl, J. Chem. Phys. 117, 1962 (2002).

${ }^{19}$ Z. Smedarchina, W. Siebrand, and M. Z. Zgierski, J. Chem. Phys. 103, 5326 (1995).

${ }^{20}$ K. Liu, J. D. Cruzan, and R. J. Saykally, Science 271, 929 (1996).

${ }^{21}$ F. N. Keutsch and R. J. Saykally, Proc. Natl. Acad. Sci. U. S. A. 98, 10533 (2001).

${ }^{22}$ X.-G. Wang and T. Carrington, J. Chem. Phys. 148, 074108 (2018).

${ }^{23}$ C. Leforestier, K. Szalewicz, and A. van der Avoird, J. Chem. Phys. 137, 014305 (2012).

${ }^{24}$ S. C. Althorpe and D. C. Clary, J. Chem. Phys. 102, 4390 (1995).

${ }^{25}$ T. R. Walsh and D. J. Wales, J. Chem. Soc., Faraday Trans. 92, 2505 (1996).

${ }^{26}$ A. van der Avoird, E. H. T. Olthof, and P. E. S. Wormer, J. Chem. Phys. 105, 8034 (1996).

${ }^{27}$ D. Sabo, Z. Bačić, T. Bürgi, and S. Leutwyler, Chem. Phys. Lett. 244, 283 (1995).

${ }^{28}$ D. Blume and K. B. Whaley, J. Chem. Phys. 112, 2218 (2000).

${ }^{29}$ T. Taketsugu and D. J. Wales, Mol. Phys. 100, 2793 (2002).

${ }^{30}$ J. O. Richardson, S. C. Althorpe, and D. J. Wales, J. Chem. Phys. 135, 124109 (2011).

${ }^{31}$ J. K. Gregory and D. C. Clary, J. Chem. Phys. 102, 7817 (1995).

${ }^{32}$ M. Quack and M. A. Suhm, Chem. Phys. Lett. 234, 71 (1995).

${ }^{33}$ M. Takahashi, Y. Watanabe, T. Taketsugu, and D. J. Wales, J. Chem. Phys. 123, 044302 (2005).

${ }^{34}$ L. H. Coudert and J. T. Hougen, J. Mol. Spectrosc. 130, 86 (1988).

${ }^{35}$ J. O. Richardson, C. Pérez, S. Lobsiger, A. A. Reid, B. Temelso, G. C. Shields, Z. Kisiel, D. J. Wales, B. H. Pate, and S. C. Althorpe, Science 351, 1310 (2016).

${ }^{36}$ J. O. Richardson, D. J. Wales, S. C. Althorpe, R. P. McLaughlin, M. R. Viant, O. Shih, and R. J. Saykally, J. Phys. Chem. A 117, 6960 (2013).

${ }^{37}$ M. T. Cvitaš and J. O. Richardson, Phys. Chem. Chem. Phys. 22, 1035 (2019).

${ }^{38}$ E. Mátyus, D. J. Wales, and S. C. Althorpe, J. Chem. Phys. 144, 114108 (2016).

${ }^{39}$ C. L. Vaillant, D. J. Wales, and S. C. Althorpe, J. Chem. Phys. 148, 234102 (2018).

${ }^{40}$ C. L. Vaillant, D. J. Wales, and S. C. Althorpe, J. Phys. Chem. Lett. 10, 7300 (2019).

${ }^{41}$ A. I. Vainshtein, V. I. Zakharov, V. A. Novikov, and M. A. Shifman, Sov. Phys. Uspekhi 25, 195 (1982); also in Instantons in Gauge Theories, edited by M. Shifman (World Scientific, Singapore, 1994), p. 468.

${ }^{42}$ R. P. Feynman and A. R. Hibbs, Quantum Mechanics and Path Integrals (McGraw-Hill, New York, 1965).

${ }^{43}$ G. V. Mil'nikov and H. Nakamura, J. Chem. Phys. 115, 6881 (2001).

${ }^{44}$ H. Nakamura and G. Mil'nikov, Quantum Mechanical Tunneling in Chemical Physics (CRC Press, Boca Raton, FL, 2013).

${ }^{45}$ Z. Smedarchina, W. Siebrand, and A. Fernández-Ramos, J. Chem. Phys. 137, 224105 (2012).

${ }^{46}$ W. Siebrand, Z. Smedarchina, M. Z. Zgierski, and A. Fernández-Ramos, Int. Rev. Phys. Chem. 18, 5 (1999).

${ }^{47}$ J. O. Richardson and S. C. Althorpe, J. Chem. Phys. 134, 054109 (2011).

${ }^{48}$ H. Kleinert, Path Integrals in Quantum Mechanics, Statistics, Polymer Physics and Financial Markets, 5th ed. (World Scientific, Singapore, 2009). 
${ }^{49}$ L. S. Schulman, Techniques and Applications of Path Integration (Wiley, 1981).

${ }^{50}$ T. Kawatsu and S. Miura, J. Chem. Phys. 141, 024101 (2014).

${ }^{51}$ W. H. Miller, J. Chem. Phys. 62, 1899 (1975).

${ }^{52}$ J. O. Richardson, Int. Rev. Phys. Chem. 37, 171 (2018).

${ }^{53}$ J. O. Richardson, Faraday Discuss. 195, 49 (2016).

${ }^{54}$ S. R. McConnell, A. Löhle, and J. Kästner, J. Chem. Phys. 146, 074105 (2017).

${ }^{55}$ J. B. Rommel and J. Kästner, J. Chem. Phys. 134, 184107 (2011).

${ }^{56}$ A. Löhle and J. Kästner, J. Chem. Theory Comput. 14, 5489 (2018).

${ }^{57}$ P. Winter and J. O. Richardson, J. Chem. Theory Comput. 15, 2816 (2019).

${ }^{58}$ C. Vaillant and M. T. Cvitaš, Phys. Chem. Chem. Phys. 20, 26809 (2018).

${ }^{59}$ J. Nocedal, Math. Comput. 35, 773 (1980).

${ }^{60}$ D. C. Liu and J. Nocedal, Math. Program. 45, 503 (1989).

${ }^{61}$ M. T. Cvitaš, J. Chem. Theory Comput. 14, 1487 (2018).

${ }^{62}$ S. K. Burger and W. Yang, J. Chem. Phys. 124, 054109 (2006).

${ }^{63} \mathrm{M}$. T. Cvitaš and J. O. Richardson, in Molecular Spectroscopy and Quantum Dynamics, edited by R. Marquardt and M. Quack (Elsevier, 2020), Chap. 10.

${ }^{64}$ D. M. Einarsdóttir, A. Arnaldsson, F. Óskarsson, and H. Jónsson, in Applied Parallel and Scientific Computing: 10th International Conference, PARA 2010,
Lecture Notes in Computer Science Vol. 7134, edited by K. Jónasson (SpringerVerlag, Berlin, 2012), pp. 45-55.

${ }^{65}$ H. Goldstein, C. Poole, and J. Safko, Classical Mechanics, 3rd ed. (AddisonWesley, San Francisco, 2002).

${ }^{66}$ P. R. Bunker and P. Jensen, Molecular Symmetry and Spectroscopy, 2nd ed. (NRC Research Press, Ottawa, 2006).

${ }^{67}$ W. H. Press, S. A. Teukolsky, W. T. Vetterling, and B. P. Flannery, Numerical Recipes: The Art of Scientific Computing, 3rd ed. (Cambridge University Press, Cambridge, 2007).

${ }^{68}$ T. Baba, T. Tanaka, I. Morino, K. M. T. Yamada, and K. Tanaka, J. Chem. Phys. 110, 4131 (1999).

${ }^{69}$ S. K. Reddy, S. C. Straight, P. Bajaj, C. Huy Pham, M. Riera, D. R. Moberg, M. A. Morales, C. Knight, A. W. Götz, and F. Paesani, J. Chem. Phys. 145, 194504 (2016).

${ }^{70}$ U. Góra, W. Cencek, R. Podeszwa, A. van der Avoird, and K. Szalewicz, J. Chem. Phys. 140, 194101 (2014).

${ }^{71}$ Y. Wang, X. Huang, B. C. Shepler, B. J. Braams, and J. M. Bowman, J. Chem. Phys. 134, 094509 (2011).

${ }^{72}$ Y. Wang, B. C. Shepler, B. J. Braams, and J. M. Bowman, J. Chem. Phys. 131, 054511 (2009).

${ }^{73}$ G. V. Mil'nikov and H. Nakamura, J. Chem. Phys. 122, 124311 (2005). 